\title{
1 An Epigenetic Priming Mechanism Mediated by Nutrient Sensing Regulates 2 Transcriptional Output
}

3

4 Natalia Stec ${ }^{1}$, Katja Doerfel ${ }^{1}$, Kelly Hills-Muckey ${ }^{1}$, Victoria M. Ettorre ${ }^{2}$, Sevinc Ercan ${ }^{2}$, Wolfgang 5 Keil $^{3^{*}}$, C. M. Hammell ${ }^{1^{*}}$

6

$7 \quad{ }^{1}$ Cold Spring Harbor Laboratory, Cold Spring Harbor, New York, 11724, USA

8 2New York University, Center for Genomics and Systems Biology, New York, New York, 10003, 9 USA

10 'Laboratoire Physico Chimie Curie, Institut Curie, PSL Research University, CNRS UMR168, 26

11 rue d'Ulm, 75248 Paris Cedex 05, France

12

13

14 *Correspondence: wolfgang.keil@curie.fr (W.K.), chammell@cshl.edu (C.M.H.) 


\section{Summary}

16 While precise tuning of gene expression levels is critical for most developmental 17 pathways, the mechanisms by which the transcriptional output of dosage-sensitive 18 molecules is established or modulated by the environment remain poorly understood. 19 Here, we provide a mechanistic framework for how the conserved transcription factor 20 BLMP-1/Blimp1 operates as a pioneer factor to decompact chromatin near its target 21 loci hours before transcriptional activation and by doing so, regulates both the duration

22 and amplitude of subsequent target gene transcription. This priming mechanism is 23 genetically separable from the mechanisms that establish the timing of transcriptional 24 induction and functions to canalize aspects of cell-fate specification, animal size 25 regulation, and molting. A key feature of the BLMP-1-dependent transcriptional 26 priming mechanism is that chromatin decompaction is initially established during 27 embryogenesis and maintained throughout larval development by nutrient sensing. 28 This anticipatory mechanism integrates transcriptional output with environmental 29 conditions and is essential for resuming normal temporal patterning after animals exit 30 nutrient-mediated developmental arrests. 


\section{INTRODUCTION}

33 Many developmental systems produce invariable phenotypes despite being confronted

34 with perturbations, such as fluctuations in temperature and nutrient availability. How this

35 robustness emerges from developmental gene regulatory networks (GRNs) and how aspects of

36 precision and flexibility are balanced to generate robust developmental programs is a major open

37 question in developmental biology. C. elegans serves as a unique genetic model for unraveling

38 the mechanisms underlying precision and robustness of gene regulation during development.

39 This is in part because the GRNs that control the essentially invariant cell lineage can generate

40 the identical developmental outcomes in the context of diverse stochastic, environmental or

41 genetic perturbations. A striking example of such a GRN is the so-called heterochronic pathway

42 which controls the timing, sequence and synchrony of stage-specific patterns of cell divisions

43 throughout post-embryonic development (Ambros and Horvitz, 1984). Analysis of this GRN

44 indicates that it generates modular patterns of gene expression that occur in the context of the

45 repetitive post-embryonic molting cycle (Ambros and Horvitz, 1984) which, in turn, demarcates

46 the four successive larval stages ( $L 1-L 4)$. Transitions from one temporal program to the next

47 occur through the expression of distinct microRNAs (miRNAs) that down-regulate the expression

48 of their temporal selector gene targets in a strict dosage-sensitive manner. Ectopic or abnormally

49 lower or higher doses of heterochronic genes (e.g. miRNAs) result in the wholesale skipping or

50 reiteration of stage-specific developmental programs (Rougvie and Moss, 2013, Feinbaum and

51 Ambros, 1999; Li et al., 2005; Perales et al., 2014; Van Wynsberghe and Pasquinelli, 2014). While

52 the regulatory interactions that establish the sequence of stage-specific events are known, we

53 have very little understanding of how the precise timing or levels of gene expression within this

54 GRN are established and how both of these are buffered against perturbations to achieve robust

55 temporal development of the animal. 
57 features associated with global gene expression patterns in developing C. elegans larva

58 (Hendriks et al., 2014; Kim et al., 2013). These studies indicate that between 10 to $20 \%$ of the

59 post-embryonic transcriptome exhibits highly reproducible periodic expression patterns.

60 Periodic transcription occurs in a variety of environmental conditions and is independent of life

61 history, indicating that it is under tight genetic control. Importantly, the transcriptional rhythm

62 follows the cycle of post-embryonic molting, a process that demarcates patterns of stage-

63 specific developmental programs. Under various environmental conditions that modulate overall

64 developmental pace, the timing of transcription onset scales accordingly, such that the phase of

65 transcription onset within the molting cycle is preserved (Hendriks et al., 2014; Kim et al., 2013).

66 Currently, it is not known how these transcriptional rhythms are generated, how they are

67 integrated into the execution of stage-specific cellular programs, and how environmental or

68 internal cues modulate features of these transcriptional patterns to achieve robust progression

69 through developmental programs, even after prolonged developmental arrests.

70 The heterochronic GRN is integrated with global aspects of transcription as each of the

71 miRNAs in this pathway exhibits an oscillatory expression. The most promising candidate gene

72 that integrates the rhythm of $C$. elegans post-embryonic molting to changes in repetative

73 transcriptional patterns is lin-42. The lin-42 gene encodes the $C$. elegans ortholog of PERIOD/Per

74 proteins that are an essential component of the circadian clock in mice, Drosophila and humans

75 (Hurley et al., 2016; Jeon et al., 1999; Monsalve et al., 2011). Similar to PERIOD, LIN-42 functions

76 as a transcriptional repressor and exhibits a cyclical expression pattern In contrast to the

77 temperature-invariant approximate 24-hour periodicity of Period, lin-42 transcription is tied to

78 the larval molting cycle, and thus, not temperature compensated (Monsalve et al., 2011; Perales

79 et al. 2014). Hypomorphic mutations in lin-42 cause animals to prematurely execute adult-

80 specific gene expression patterns in the L4 stage of larval development and these phenotypes 
81 are caused by the cumulative, premature over-expression of multiple heterochronic miRNAs

82 (McCulloch and Rougvie, 2014; Perales et al., 2014; Van Wynsberghe and Pasquinelli, 2014). In

83 addition to these temporal patterning phenotypes, lin-42 mutants also exhibit lengthened and

84 irregular molting cycles (Monsalve et al. 2011; Tennessen et al., 2010). The mechanisms by which

85 LIN-42 negatively regulates heterochronic miRNA transcriptional output remain unknown.

86 Furthermore, it is not known if other regulatory components exist that antagonize LIN-42

87 functions to ensure normal transcriptional regulation of developmental timing genes.

88 Here, we leverage the developmental phenotypes of lin-42 mutations to identify BLMP-1

89 and ELT-3 as conserved transcription factors (TFs) that modulate the transcriptional output of

90 many cyclically expressed genes to provide developmental robustness. We demonstrate that

91 these TFs coordinate a plethora of post-embryonic developmental programs and we present a

92 unique role for BLMP-1 in transcriptional priming that involves chromatin decompaction of target

93 loci. Specifically, we show that BLMP-1 activity modulates the duration and amplitude of

94 transcription of its targets without altering the timing of expression within the repetitive

95 transcriptional cycle. We hypothesize that BLMP-1 functions specifically to assure proper

96 transcriptional output in diverse environmental conditions. Consistent with this model, we

97 demonstrate that the BLMP-1-dependent remodeling of chromatin is regulated by nutritional

98 inputs and becomes essential when animals resume development after nutrient-mediated

99 developmental arrests. 


\section{RESULTS}

102 blmp-1 and elt-3 Function in a partially redundant manner to control temporal patterning 103 during larval development.

104 To identify genes that antagonize the function of LIN-42 to modulate transcriptional output, we

105 took advantage of a highly penetrant phenotype of the lin-42 loss of function allele lin-42(n0189)

106 - the precocious expression of an adult-specific col-19::GFP reporter in both seam and syncytial

107 hyp7 cells of the hypodermis (Figure 1A) (Abrahante et al., 1998). We used bacterial-mediated

108 RNAi to deplete the expression of individual C. elegans TFs in lin-42(If) animals and then scored

109 col-19::GFP expression in $F_{1}$ progeny. The most reproducible suppressors of lin-42 precocious

110 phenotypes were bacterial RNAi clones expressing dsRNAs that target the blmp-1 and elt-3 loci

111 (data not shown). We validated these findings with null alleles of these genes $(b / m p-1(0)$ and elt-

$1123(0)$ ) which revealed a redundancy between b/mp-1 and elt-3 that is distributed differentially

113 across hypodermal cell types (Figure $1 \mathrm{~A}$ and B). Specifically, removal of blmp-1 activity in lin-

114 42(If) mutants completely suppresses the precocious col-19::GFP expression in the lateral seam

115 cells of L4-staged animals and only partially prevents early expression in hyp7 cells (Figure 1A

116 and B). lin-42(If); elt-3(0) mutants were only partially suppressed for the precocious col-19::GFP

117 expression phenotypes. In contrast, combining blmp-1(0) and elt-3(0) mutations with lin-42(If)

118 mutations completely alleviated precocious col-19::GFP expression in both the seam and hyp7

119 cells of a majority of L4-staged animals (Figure 1A and B). This cell-type specific redundancy is

120 likely due to the partially overlapping expression patterns of these TFs in hypodermal cells

121 throughout larval development and indicate that BLMP-1 and ELT-3 are required for lin-42(If)

122 phenotypes(Cao et al., 2017) (Figure 1C).

123 Because many of the developmental phenotypes associated with lin-42 mutations are due

124 to elevated expression of heterochronic miRNAs, we sought to determine if the genetic 125 relationships between blmp-1, elt-3 and lin-42 are mediated at the level of transcription. A let$1267::$ GFP-pest reporter is expressed in the lateral seam cells of L3 and L4-staged wild-type animals 
127 in a pulsatile fashion (once per stage). lin-42(If) animals maintain pulsatile expression patterns

128 but the amplitude of let-7::GFP-pest expression is elevated in lateral seam cells (Perales et al.

129 2014). Consistent with blmp-1 functioning up-stream of lin-42 to control let-7 transcriptional 130 output, let-7::GFP-pest expression in lin-42(If); blmp-1(RNAi) animals was reduced to almost wild-

131 type expression levels. Furthermore, let-7::GFP-pest expression in wild-type blmp-1-(RNAi)

132 animals is reduced compared to control RNAi conditions consistent with a normal function of

133 BLMP-1 in modulating transcriptional output of this miRNA. RNAi depletion of elt-3 expression did

134 not alter let-7::GFP expression in L4-staged wild-type or lin-42(If) animals (data not shown).

135 Previous analysis of blmp-1(0) mutations indicate that BLMP-1 plays an important role in

136 terminal cell fate specification of the hypodermis (Horn et al., 2014). We sought to determine if

137 blmp-1 functions earlier in temporal cell fate specification when cells are proliferating. To this end,

138 we assayed genetic interactions between blmp-1 and alg-1, the latter encoding one of the two 139 core miRISC argonaute components. alg-1(0) mutants ineffectively process most miRNAs

140 (including heterochronic microRNAs) and exhibit relatively mild temporal patterning defects that

141 resemble those associated with hypomorphic alleles of let-7 (Hammell et al. 2009). To assay

142 interactions, we quantified the number of seam cells at the mid L4 stage in wild-type or alg-1(0)

143 mutant animals in conditions where we reduced blmp-1 and/or elt-3 activity using RNAi. In wild-

144 type animals, lateral seam cells exhibit highly reproducible cell division patterns that occur at 145 specific stages of larval development (Figure 1E) (Sulston and Horvitz, 1977). Defects in this 146 lineage can be monitored by counting seam cell numbers in the L4 larval stage. Depletion of elt1473 or blmp-1 in wild-type animals resulted in the normal number of seam cells in the L4 stage. We 148 observed a significant increase in the number of SCM::GFP(+) seam cells in alg-1(0); blmp-1 149 RNAi animals (Figure 1F and G). This expansion of lateral seam cell number resembles an 150 inappropriate reiteration of earlier, L2-specific proliferative cell division programs (Figure 1E).

151 Depletion of blmp-1 in other mildly reiterative heterochronic mutants also resulted in similar 152 enhancement of cell lineage and col-19::GFP expression phenotypes (Figure S1). elt-3 RNAi did 
153 not significantly alter lateral seam cell numbers in alg-1(0) animals or enhance alg-1(0); blmp-

154 1(RNAi) phenotypes. This indicates that BLMP-1 functions early in larval development to control

155 temporal cell fates in the hypodermis.

blmp-1 and elt-3 regulate multiple dosage-dependent developmental processes

158 Development of $C$. elegans larva results in a fixed number of cells and a stereotypical size under

159 standard growth conditions. Mutations in a number of pathways (including nutrient signaling,

160 TGF $\beta$ and MAP kinase pathways) or in structural components of the cuticle alter organismal size

161 (Tuck, 2014). A shared feature of these regulatory and structural systems is that they function in

162 a dose-sensitive manner wherein changes in the expression levels of individual elements within

163 these pathways or components result in changes in animal morphology. As shown in Figure 2A,

164 blmp-1(0); elt-3(0) animals exhibit a severe dumpy (dpy) phenotype. The dpy phenotype of blmp-

165 1(0); elt-3(0) mutants manifests during post-embryonic development as freshly hatched blmp-

166 1(0); elt-3(0) larvae are indistinguishable from wild-type animals (data not shown). blmp-1(0)

167 single mutants exhibit less severe alterations in size and elt-3(0) animals are structurally normal

168 (Figure $2 \mathrm{~A}$ and $\mathrm{B}$ ). These results demonstrate that in addition to modulating aspects of temporal

169 cell-fate specification, blmp-1 and elt-3 cooperate to control animal morphology likely through the

170 co-regulation of hypodermally expressed targets.

171 A majority of blmp-1(0); elt-3(0) animals precociously die two days after they reach

172 adulthood due to the internal hatching of fertilized embryos (Figure 2C). Examination of dying

173 blmp-1(0); elt-3(0) animals revealed that most are trapped in a partially shed cuticle (Figure 2D).

174 Lethality is preceded by a reduction in movement and pharyngeal pumping approximately 8-10

175 hours after the L4-to-adult transition; a phenotype shared with other mutants that fail to post-

176 transcriptionally down-regulate the adult expression of key genes involved in larval molting (Hayes

177 et al., 2006). To determine if blmp-1(0); elt-3(0) animals inappropriately re-initiate molting

178 programs during adulthood, we directly monitored the expression of a molting-specific GFP-pest 
179 transcriptional reporter, mlt-10::GFP-pest, in wild-type, single and double mutant animals. . Wild

180 type animals never expressed mlt-10::GFP as adults $(\mathrm{n}=100)$ while a majority of blmp-1(0); elt-

$1813(0)$ animals re-expressed the mlt-10::GFP-pest reporter (54\%; $\mathrm{n}=155)$ as adults (Figure 2E).

182 blmp-1(0) single mutants also express mlt-10::GFP-pest within the same time period but with a

183 much lower penetrance $(18 \%, \mathrm{n}=137)$, consistent with previous observations using blmp-

184 1(RNAi) (Frand et al., 2005). These data indicate that blmp-1 and elt-3 function redundantly to

185 halt the behavioral and physiological molting programs once animals transition into adulthood.

186 Development of the vulval structure in the $L 3$ stage requires the integration of a number

187 of interconnected signaling pathways that establish specific cell fates in vulval precursor cells

188 (Figure 2F). One of the key initiating components in this process is let-60, encoding the C. elegans

189 ortholog of the human Ras oncogene. Continued let-60 activation commits vulval precursor cells

190 (VPCs) to a $1^{\circ}$ vulval cell fate specification pattern (Figure 2F). The induction of this fate

191 specification is sensitive to the level of let-60 activity (Beitel et al., 1990; Han and Sternberg,

192 1990). For instance, the semi-dominant let-60(n1046gf) allele can induce ectopic vulval

193 protrusions on the ventral surface (multi-vulva; muv) in $18 \%$ of animals harboring a single copy of

194 this mutation (Figure $2 \mathrm{G}$ and $\mathrm{H}$ ). We used this sensitized genetic context to determine whether

$195 \mathrm{blmp-1(0)}$ and/or elt-3(0) mutations (or the combination of these mutations) leads to an elevation

196 in VPC induction in let-60(n1046)/+ animals. Lack of blmp-1 activity significantly increased the

197 penetrance and expressivity of the muv phenotype in let-60(n1046)/+ animals (Figure G and H).

198 elt-3(0) mutations did not contribute to let-60-dependent phenotypes consistent with a lack of 199 significant ELT-3 expression in these cell types (Figure 3G) (Cao et al., 2017). Together, these 200 results indicate that $\mathrm{b} / \mathrm{mp}-1$ and elt-3 function in a partially redundant manner to regulate a 201 multitude of developmental aspects, from animal morphology, to behavior to cell fate specification. 
204 Our observations that blmp-1 and elt-3 genetically interact with lin-42 to control the transcription 205 of cyclically expressed miRNAs (e.g. let-7, Figure 1D) and that blmp-1(0); elt-3(0) double mutants 206 exhibit pleiotropic defects led us to hypothesize that these TFs may function more broadly to 207 control dynamic gene expression. To define the full repertoire of BLMP-1 and ELT-3 208 transcriptional targets, we complemented L1-staged BLMP-1 and ELT-3 ChIP-seq data obtained 209 in the modENCODE project with additional ChIP-seq experiments performed in extracts made 210 from L2-, L3-, and L4-staged animals (Araya et al., 2014; Niu et al., 2011). Analysis of these

211 combined data indicates that the regions bound by BLMP-1 in the first larval stage are also bound

212 in subsequent larval stages. Furthermore, the binding sites of BLMP-1 in the L1-L4 stages 213 significantly overlap with regions also bound by ELT-3 in the L1 stage (Figure 3A). A majority of

214 BLMP-1 peaks are enriched within 500bps upstream of the transcriptional start site (TSS) but the 215 enrichment also extends to 5 ' intergenic regions with a sharp drop-off approximately $3 \mathrm{~kb}$ upstream 216 of gene bodies of both protein coding and miRNA genes (Figure 3B and C). Peaks from all 217 datasets were assigned to the nearest proximal gene (both coding and non-coding) using these 218 parameters (Table S1).

219 To characterize the relationship between BLMP-1, ELT-3 and their targets in more detail, 220 we initially focused our analysis on protein coding genes whose expression can be reliably 221 detected in developing larvae. Our analysis employed multiple, high-resolution, and genome-wide 222 time course analyses of gene expression that parsed the larval developmental transcriptome into 223 distinct temporal expression classes (Hendriks et al., 2014; Kim et al., 2013). Both studies 224 identified significant, overlapping sets of transcripts (between $12 \%$ and $18.9 \%$ of the 225 transcriptome) that exhibit cyclical expression patterns. Hendriks et al. classified two types of 226 dynamic expression: a first class of transcripts that exhibit an oscillatory or cyclical patterns (osc) 227 that are tied to the molting cycle (osc; $18.9 \% ; 2,718$ of 14,378 total genes) or a second class of 228 genes whose expression increases monotonically throughout larval development (rising; 14.2\%) 229 (Figure 3D). To compare how BLMP-1 and ELT-3 targets may be distinguished from the targets 
230 of other TFs, we analyzed additional publicly available ChIP-seq data sets obtained by the

231 modENCODE project. This data included the targets of 170 additional C. elegans TFs that

232 represent diverse classes of TFs (Araya et al., 2014; Niu et al., 2011). Using the same target

233 assignment criteria as above, we found that most TFs are associated with a limited number of

234 target genes that exhibit a rising expression program (median targets bound $=21$ per TF;

235 interquartile range $=9$ to 54$)$ (Figure 3E; Table S2). This relationship was also conserved for the

236 associations between bulk TFs and the oscillatory class of target genes (median of 43 targets per

$237 \mathrm{TF}$; interquartile range $=18$ to 97 ). Surprisingly, we found that a limited subset of 39 TFs is

238 disproportionally associated with a large fraction of the oscillatory class of genes (Figure 3E).

239 Primary amongst this unique group of 39 TFs were BLMP-1 and ELT-3 (with 1124 and 738 of the

2402718 cyclically expressed targets bound per TF, respectively) (Figure 3E). We performed a similar

241 analysis of the Kim et al. RNA-seq data to determine if ELT-3 or BLMP-1 binding sites are also

242 enriched in the promoters of cyclically expressed transcripts as defined by their temporal

243 classifications. Our analysis found that BLMP-1 binding sites, but not ELT-3 binding sites, are

244 enriched in the promoters of each cyclically expressed genes class and de-enriched for those that

245 exhibit monotonic expression patterns (Figure S3 and Table S3).

246 One robust feature of cyclically expressed mRNAs is that the timing of expression within

247 each larval stage occurs in the same phase of each transcriptional cycle. This expression/phase

248 relationship is preserved even when animals are grown at lower or higher temperatures which

249 lengthens or shortens the periodicity of these patterns, respectively (Hendriks et al., 2014; Kim et

250 al., 2013). Because BLMP-1 and ELT-3 are constitutively expressed through each larval stage

251 (Figure 3F), we aimed to determine if the subset of BLMP-1 and ELT-3 targets that exhibit cyclical

252 expression patterns were concentrated in any particular phase of expression during each larval

253 stage. To compare these patterns, we plotted the peak phase (in degrees/radians of a repetitive

254 cycle) and expression level ( $\log _{2}$ change in expression as an increasing radius from the center)

255 for each of the cyclically expressed transcripts on a radial chart (Figure 3G). ELT-3 and BLMP-1 
256 targets were distributed across each sector of the repetitive temporal pattern and were not 257 statistically enriched for any phase of expression. The distributed associations of these TFs and 258 their varied targets combined with the constitutive expression of BLMP-1 and ELT-3 throughout 259 each larval stage suggest that BLMP-1 and ELT-3 are associated with their targets throughout 260 the transcriptional cycle regardless of transcriptional class of the target gene or their timing of 261 expression.

263 Conserved sequences of the upstream regulatory regions of lin-4 are necessary and 264 sufficient for high amplitude expression in hypodermal tissues

265 Many of the heterochronic phenotypes associated with a null mutant of lin-4, lin-4(e912), can be 266 partially rescued by a high-copy transformation of a $693 \mathrm{bp}$ genomic fragment that contains 267 approximately 500 bp of upstream putative regulatory sequence (Lee et al., 1993). Analysis of the 268 RNAs that are produced from the lin-4 locus indicate transcription of pri-lin-4 is initiated at atleast 269 five TSS upstream of the lin-4 gene (Bracht et al., 2010). Of these TSSs, three occur upstream of 270 the minimal rescuing fragment, suggesting that additional regulatory information may be required 271 to control normal aspects of lin-4 transcription. These upstream regions are also associated with 272 the active histone modifications H3K4me3 and open chromatin as measured by ATAC-seq 273 assays (Figure 4A) (Daugherty et al., 2017; Janes et al., 2018). We compared the syntenic 274 sequences of five additional nematode species to determine if regions of the F59G1.4 ninth intron 275 were conserved through evolutionary selection. This analysis revealed at least three discrete 276 regions upstream of each lin-4 gene that were disproportionately conserved when compared to 277 similarly sized intronic and intragenic regions (Conserved Elements (CE) A, C and D). 278 Examination of our ChIP-seq data indicate that each of these regions is enriched for BLMP-1 279 binding (Figure 4A, Table S1). We did not detect a strong enrichment of ELT-3 bound to the 280 putative lin-4 regulatory sequences though multiple predicted ELT-3 consensus binding site 281 sequences are found in this region (Figure 4A). 
To test the functional relevance of these elements, we constructed a transcriptional GFP-

283 pest reporter that harbors a SL2 splice acceptor sequence directly upstream of the GFP open

284 reading frame (Figure 4A; Full Length $(F L)$ ). This feature enables each reporter to generate a

285 single, defined mRNA transcript from each of the various candidate TSSs. This reporter is robustly

286 expressed in a variety of cell types including the hypodermis, pharynx, body wall muscle, and

287 ventral neurons on the surface of larva. Expression of the lin-4::GFP-pest reporter occurs once

288 per larval stage (similar to other characterized transcriptional reporters) in these cell types

289 indicating that this sequence contains the information to drive temporally and spatially restricted

290 expression in multiple cell lineages. To characterize the temporal expression patterns in detail,

291 we measured the levels of lin-4::GFP-pest expression in lateral seam cells at each of the

292 morphologically defined stages of L4 development (Mok et al., 2015). These experiments

293 revealed that GFP-pest expression is highly pulsatile with expression peaking at the L4.5 and

294 L4.6 stages (Figure 4B and C). Expression in other cell types (neuronal, pharynx, muscle and

295 hyp7 cells) also peak during these larval substages with different expression trajectories (Data

296 not shown). Specifically, muscle cells exhibit a broader (longer) transcriptional pulse compared to

297 those observed in seam cells. Expression in hyp7 and neuronal cell types exhibit a shorter pulse

298 of transcription either muscle or seam cells (Figure 4B).

299 We then compared the expression patterns we observed using the full-length construct to

300 previously published reporters or variants of the full-length reporter that lack individual or

301 combinations of the conserved sequence elements outlined above. Each of the reporters express

302 pulses of GFP-pest expression in similar cell types as the full-length version (Bracht et al., 2010;

303 Perales et al., 2014). However, peak expression in the hypodermal cells was typically 5-20-fold

304 higher from the full-length reporter compared to the other derivative reporter genes (Figure 4D).

305 Deletion analyses of the full-length construct indicate that CE A, harboring sequences that bind

306 BLMP-1 in vivo, are primarily responsible for this increased transcriptional output as reporters that 
307 lack this element exhibit residual expression to a similar level as the minimal rescuing fragment 308 (Figure 4D and E). Using the ChIP-seq data sets for BLMP-1::GFP, we identified a consensus sequence for

310 BLMP-1 that is enriched in the CE A regulatory sequence (Figure 4A and C). A recombinant GST 311 translational fusion of the five BLMP-1 zinc fingers (ZnFs), GST-BLMP-1(ZnFs), but not GST 312 alone bound several sequences found in CE A element that conform to this consensus. This 313 binding was diminished by the addition of cold competitor DNA harboring consensus BLMP-1

314 binding sites but not by a similar sized, non-specific target DNA (Figure 4G). To determine if these 315 elements function as a transcriptional enhancer, we cloned a 138bp fragment from the CE A 316 element, called minimal $\mathrm{CE}$ ( $\operatorname{minCE}$ ) (Figure 4A and $\mathrm{H}$ ), into the 5 ' regulatory sequences of the 317 gst-5 gene encoding the C. elegans ortholog of human HPGDS (hematopoietic prostaglandin D 318 synthetase). RNA-seq experiments demonstrate that gst-5 mRNAs are expressed throughout 319 larval development in a variety of tissues (including the hypodermis) and exhibit a non-cyclical or

320 flat expression pattern (Cao et al., 2017; Hendriks et al., 2014; Kim et al., 2013). A transcriptional

321 reporter bearing $2.9 \mathrm{~kb}$ of the gst-5 upstream regulatory sequences drives a low level GFP-pest

322 expression in a number of larval cell types including the pharynx, intestine, neurons and 323 hypodermis and this expression pattern remained below the threshold of imaging during normal 324 development (Figure $4 \mathrm{H})$. In contrast, the transcriptional reporter that included two copies of the $325 \operatorname{minCE}$ in the reverse orientation (reversed compared to the orientation within the lin-4 enhancer 326 element) drove high amplitude, periodic expression of GFP-pest exclusively in hypodermal cells 327 (Figure $4 \mathrm{H})$. Expression of the (2xminCE)gst-5::GFP-pest reporter in blmp-1(0) mutant animals 328 resembled the expression of the simple, gst-5::GFP-pest reporter in wild-type animals (Figure 329 S4), consistent with the hypothesis that this minimal sequence from the lin-4 upstream region 330 functions as both a spatial and temporal enhancer.

331

332 BLMP-1 controls the transcriptional output of its targets 
333 A large fraction of BLMP-1 and ELT-3 targets are expressed in a cyclical fashion (approximately $33426 \%$ and $23 \%$, respectively) (Figure 3D and Table S2). We reasoned that targets that exhibit this 335 highly dynamic form of gene expression could provide an opportunity to determine the effects of 336 BLMP-1 and/or ELT-3 binding on transcriptional dynamics in more detail. To quantify changes in 337 expression dynamics, we obtained or constructed integrated transcriptional GFP-pest reporters 338 that fit two criteria. First, we identified candidate genes that are predicted to be targets of BLMP3391 and/or ELT-3 as measured by ChIP-seq (Figure 3). Second, we selected genes that are 340 predicted (by RNA-seq experiments) to be dynamically expressed in hypodermal tissues (Cao et 341 al., 2017). We therefore constructed or obtained multi-copy integrated transcriptional GFP-pest 342 reporters for $(2 x \operatorname{minCE})$ gst-5, moe-3, mlt-10, and ZK180.5 and a single copy version of a 343 transcriptional GFP-pest reporter for C02E7.6 integrated on chromosome II. To characterize the 344 temporal expression patterns of these reporters, we assembled a series of measurements that 345 spanned the morphologically defined stages of $L 4$ development. As shown in Figures $5 A-C$, each 346 reporter is expressed in a pulsatile expression pattern with (2xminCE)gst-5 and C02E7.6 347 reporters exhibiting a peak of expression in the mid-L4 stage, and the moe-3 transcriptional 348 reporter reaching maximal amplitude near the end. We next aimed to determine how mutations 349 in blmp-1 and/or elt-3 altered these expression patterns. GFP-pest expression levels in seam 350 cells were then quantified in each genetic context at a developmental stage where reporter 351 expression peaks in wild-type animals. As illustrated in Figure $5 \mathrm{~A}$ and $\mathrm{B}$, blmp-1(0) mutations 352 dampen the expression of both (2xminCE)gst-5 and C02E7.6 reporters by approximately 1.7 -fold. 353 In contrast, elt-3(0) mutations had little effect on the transcriptional output of either of these two 354 reporters. Furthermore, the decrease in seam cell expression for the (2xminCE)gst-5 and 355 C02E7.6 reporters was not further decreased in blmp-1(0); elt-3(0) double mutants, consistent 356 with a lack of ELT-3 binding sites in these promoters. In contrast, examination of moe-3::GFP357 pest expression in various mutant backgrounds indicate that activity of both blmp-1 and elt-3 are 358 required to maintain high transcriptional output for this gene in the hypodermis (Figure $5 \mathrm{C}$ ). 
We next sought to correlate dynamic changes in gene expression to other aspects of larval development in more detail. To accomplish this, we performed long-term, live imaging of

361 developing larvae in a microfluidic device (Keil et al., 2016). This imaging strategy enabled us to 362 continuously monitor gene expression in individual animals and also correlate these changes with

363 other cellular and developmental milestones. First, we compared overall developmental pace

364 between WT, blmp-1(0), elt-3(0) and blmp-1(0);elt-3(0) animals in the microfluidic chambers by

365 assessing the time between the successive ecdysis (cuticle shedding). While both WT and mutant

366 animals displayed variability of larval stage duration within each genotype, neither blmp-1(0) nor

367 elt-3(0) mutations (or combination of these mutations) affected the average duration to a

368 substantial degree (Figure 5D and Figure S5). However, our live imaging experiments revealed a

369 partially penetrant larval lethality phenotype, in particular in the L3 stage, that was not apparent

370 on standard, solid NGM plates.

371 The fact that the larval stage durations are largely preserved between WT and blmp-

$3721(0) / e l t-3(0)$ mutants allowed us to directly compare average quantitative temporal features of

373 transgene gene expression dynamics in each genetic background. To this end, we directly

374 monitored patterns of two GFP-pest reporters, ZK180.5::GFP-pest and mIt-10::GFP-pest, that are

375 exclusively expressed in the hypodermis (Figure 5E) and whose putative regulatory elements are

376 bound by BLMP-1 and ELT-3 (Figure 3 and Figure S6). To compare the expression patterns

377 between animals, we extracted individual animal fluorescence time course measurements and

378 rescaled them such as to align the timing of molting events of each animal (see Methods) to the

379 average WT molting times (Figure 5F). This procedure enabled us to directly compare specific

380 features between each expression trajectory including amplitude, transcriptional onset (the time

381 within each transcriptional cycle in which transcription begins), peak phase (the point within each

382 cycle in which the peak expression is reached), and the cumulative accumulation of GFP-pest

383 expression (output). Consistent with other blmp-1- or blmp-1; elt-3-dependent reporters, cyclical

384 transcription of ZK180.5::GFP-pest and mlt-10::GFP-pest was maintained throughout larval 
385 development indicating that blmp-1 (or elt-3) are not essential for generating pulsatile patterns of

386 transcription (Figure 5 F-J and Figure S6). The primary difference between the expression

387 trajectories of wild type and mutant animals was the drastic reduction in the amplitude of individual

388 traces in blmp-1(0) mutants. Surprisingly, the reduction of transcriptional output was not

389 accompanied by any substantial change in the timing of transcriptional induction (Figure $5 \mathrm{H}$ and

$390 \mathrm{~J}$ and Figure S6). Ultimately, the reduction of transcriptional output (as measured by total

391 cumulative GFP-pest expression during each pulse) correlates with a reduction in the relative

392 duration of expression in mutant animals (Figure $5 \mathrm{H}$ and $\mathrm{J}$ and Figure S6). These results indicate

393 that BLMP-1 functions to amplify the baseline pulsatile transcriptional dynamics that are likely

394 generated by other gene regulatory mechanisms.

395

396 The lin-4 locus adopts a BLMP-1-dependent, active chromatin structure in hypodermal

397 cells

398 Previous studies indicate that BLMP-1 physically and functionally interacts with multiple 399 chromatin remodeling factors (e.g., SWI/SNF subunits) and that BLMP-1 binding sites are 400 enriched in open chromatin regions (Fong et al., 2020; Daugherty et al., 2017; Janes et al., 2018).

401 We hypothesized that BLMP-1 may function to potentiate transcription through chromatin 402 remodeling near its binding sites which would potentiate the binding of additional TFs. This model 403 would account for the dependency of the diverse transcriptional targets of BLMP-1 that differ in 404 their temporal pattern of expression (phase) during the repetitive transcriptional cycle and would 405 be mechanistically similar to the activities of other TFs (including pioneer factors) that remodel 406 chromatin accessibility prior to the onset of major transcriptional induction in C. elegans (Cochella 407 and Hobert, 2012; Hsu et al., 2015; Patel and Hobert, 2017).

408 We therefore sought to measure how BLMP-1 activity may alter chromatin dynamics at a 409 specific locus with spatiotemporal resolution. We utilized an established chromosome-tagging 410 strategy that has been employed to visualize both the localization of transgenic arrays and 
411 dynamics of chromatin compaction in living animals (Cochella and Hobert, 2012; Fakhouri et al.,

412 2010; Meister et al., 2010). Transgenes containing the lin-4(FL)::mCherry-pest ORF flanked by

413 tandem copies of the bacterial lac operator (lacO) sequences were integrated to a single

414 chromosomal site and then crossed into a strain harboring a soluble, ubiquitously expressed GFP-

415 tagged lac repressor ( $\mathrm{Lacl}$ ) fusion that recognizes lacO sequences with high affinity (Patel and

416 Hobert, 2017). The enabled us to monitor the lin-4 locus in live animals while simultaneously

417 monitoring transcriptional output of the lin-4 enhancer/promoter (Figure 6A). Animals harboring

418 these transgenes exhibit two foci in each somatic nucleus indicating the stable, single integration

419 site of the lin-4::mCherry/LacO transgene. The intranuclear GFP-Lacl foci found in freshly hatched

420 L1-staged animals can be grossly categorized into two basic classes: Class 1) a punctate or "ball-

421 like" pair of GFP-Lacl foci that are found in a majority of somatic cells and Class 2) pairs of "puffed"

422 foci that are found in the nuclei of hypodermal cells (Figure 6B). This lineage-specific

423 decompaction of the lin-4 locus is surprising for multiple reasons. First, lin-4 loci appear to be

424 decompacted only in hypodermal tissues where BLMP-1 is normally expressed. Second,

425 chromatin decompaction is typically associated with active transcription in a variety of systems

426 (Dietzel et al., 2004; Tumbar et al., 1999; Yuzyuk et al., 2009) and the decompaction of the lin-4

427 loci in freshly hatched L1-stage animals precedes the transcriptional induction of lin-4 miRNAs by

428 approximately 12 hours (note a lack of $m$ Cherry expression in Figure 6B).

429 To test whether BLMP-1 expression correlates with target loci decompaction, we

430 performed two types of analyses. First, we characterized when BLMP-1 expression begins in

431 embryos. BLMP-1::GFP can be detected as early as the bean stage (approximately 365 minutes

432 post fertilization) in most developing hypodermal cells (Figure 6C). This tissue-specific expression

433 pattern remains throughout the rest of embryonic development (Figure 6C). We next aimed to

434 determine how the timing of BLMP-1 expression correlates with the decompaction level of the lin-

4354 loci by examining changes in GFP-Lacl foci. From the onset of GFP-Lacl expression to the early

436 gastrulation stage of embryogenesis, most somatic lin-4 GFP-Lacl foci are decompacted, 
consistent with a general lack of condensed chromatin and reduced histone H3 lysine 9 (H3K9)

438 methyltransferase activity in early embryos (Mutlu et al., 2019; Mutlu et al., 2018; Towbin et al.,

439 2012; Yuzyuk et al., 2009). By the end of gastrulation (approximately 330 minutes post

440 fertilization) the foci in most somatic cells are equally condensed consistent with the onset of

441 large-scale heterochromatin formation in somatic cells and a reduction in transcriptional plasticity

442 (Mutlu et al., 2019; Mutlu et al., 2018; Yuzyuk et al., 2009). At roughly the same time as the onset

443 of BLMP-1 expression in embryonic hypodermal cells, the GFP-Lacl foci begin to decompact in

444 posterior seam cells (Figure 6D). The cell type-specific decompaction of lin-4 loci in hypodermal

445 tissues is complete for a majority of wild-type animals by the end of embryogenesis while the

446 same foci in other somatic cell types remain tightly compressed (Figure 6D). GFP-Lacl foci

447 dynamics were similar in wild-type and blmp-1(0) animals during early embryogenesis. In contrast

448 to the late embryonic decompaction of lin-4 loci in wild-type animals, hypodermal GFP-Lacl foci

449 (and foci in other somatic nuclei) in blmp-1(0) mutants remained condensed (Figure 6D and E).

450 We conclude that BLMP-1 is required for decompaction of the lin-4 loci in hypodermal cells during

451 embryogenesis. Furthermore, this decompaction occurs at a point in development where lin-4

452 transcription is being actively repressed by multiple FLYWCH proteins (Ow et al. 2008).

453 We next aimed to directly monitor any dynamic changes in "puffs" sizes within the

454 transcriptional cycle of lin-4 during larval development and determine if these changes are 455 dependent on BLMP-1 activity. In head ganglion cells, derived from a cell lineage that never 456 express the lin-4pro::mCherry-pest transgene, the two lin-4pro::mCherry-pest loci are 457 constitutively compacted (Figure 6F and G). To measure changes in cells that do express lin-4 458 miRNAs, we focused our analysis on developing lateral seam cells for multiple reasons. First, 459 seam cells constitutively express BLMP-1 and dynamically express the lin-4pro::mCherry-pest 460 reporter at specific phases of each larval stage (Figure 1B and Figure 4A). Second, in contrast to 461 hyp7 syncytial cells that endoreduplicate their nuclear DNA content at each division resulting in a 462 stable ploidy of $4 \mathrm{n}$, seam cells replicate their nuclear DNA once per division at specific, defined 
463 phases of the larval molt cycle (Hedgecock and White, 1985). To ensure accuracy in both the

464 timing of lin-4::mCherry-pest expression and seam cell divisions, we specifically measured the 465 compaction status of the lin-4::mCherry-pest loci in late L3-staged animals where morphological 466 features of the vulval cell lineage, gonad morphology, and mCherry-pest expression could be 467 used to compare animals of both genotypes at similar developmental stages. We found that the 468 GFP-Lacl "puffs" size in non-transcriptionally active lateral seam cells was typically three times 469 larger than the those found in neurons (Figure 6F and G). Later, during the L3 lethargus, when 470 lin-4pro::mCherry-pest is expressed, we observed that the average size of the GFP-Lacl "puffs"

471 further increase in size (approximately an additional 2-fold when compared to non-

472 transcriptionally active seam cells) (Figure $6 \mathrm{~F}$ and $\mathrm{G}$ ). This suggests that the lin-4 locus is 473 constitutively decompacted (compared to the same locus in neuronal cells) and the process of 474 transcribing this locus further relaxes chromatin architecture. We then asked if chromatin 475 decompaction at these loci was dependent on BLMP-1 activity by examining the same features 476 in blmp-1(0) animals. A comparison of the GFP-Lacl "puff" sizes in head neurons indicates that 477 they are equally compressed in wild-type and blmp-1(0) animals (Figure 6F and G). In contrast, 478 GFP-Lacl foci, in both non-transcribing and lin-4 expressing cells, are significantly reduced in size 479 compared to those observed in stage-matched wild-type animals (Figure 6F and $\mathrm{G}$ ). These 480 experiments demonstrate that the chromatin structure of the lin-4 locus is dynamically remodeled 481 in seam cells and that BLMP-1 is required for decompaction of the lin-4 locus in hypodermal cells 482 prior to transcription in wild-type, larval animals.

484 BLMP-1 and ELT-3 are required to resume normal temporal patterning after nutrient 485 deprivation

486 Development of $C$. elegans larvae in laboratory conditions is both rapid and continuous 487 due to an abundant, controlled food supply and standardized growth conditions. In contrast, the 488 natural environment in which C. elegans larvae normally live is far more diverse and dynamic 
489 leading to a more punctuated developmental trajectory (Frezal and Felix, 2015). Acute food 490 removal induces a defined developmental diapause that can occur immediately after each larval 491 molt (Schindler et al. 2014). The diapause is part of a developmental checkpoint that is distinct 492 from dauer development and controlled by insulin signaling (Schindler et al. 2014). When the

493 insulin signaling rapidly inactivated (i.e. during acute starvation), animals finish the current larval

494 stage, arrest all somatic cell movements and divisions, and halt the expression of molting-specific 495 genes (Schindler et al., 2014).

496 To determine if global aspects of cyclical transcription are altered during acute starvation,

497 we monitored the expression of four transcriptional reporters that exhibit distinct phases of 498 expression (Figure 5). These reporters include targets of BLMP-1 and ELT-3 ((minCE)gst-5::GFP499 pest, ZK180.5::GFP-pest, and mlt-10::GFP-pest) as well as a transcriptional reporter of blmp-1, 500 since ChIP-seq data indicates that it is expressed in a cyclical expression pattern (Hendriks et al., 501 2014; Kim et al., 2013) and BLMP-1 activity regulates its own expression in an autocatalytic 502 fashion (Figure S7). We grew populations of L1-staged animals under nutrient-rich conditions for 50322 hours and then one half of each sample was allowed to continue development in nutrient 504 replete conditions while the other half was acutely starved (Figure 7A). Acute food removal results 505 in greater than $90 \%$ of transgenic animals arresting development with undivided vulval precursor 506 cells after 24 hours indicating that larval development has been paused (Schindler et al., 2014). 507 During the starvation period, we monitored GFP-pest expression in fed and starved populations. 508 In contrast to the cyclical expression of each reporter under nutrient replete conditions, GFP-pest 509 expression for each of the reporters was extinguished during starvation conditions with similar 510 kinetics (Figure 7B). This transcriptionally inactive state was maintained throughout the starvation

511 period. Re-fed animals resumed normal, cyclical expression patterns within a few hours after food 512 exposure (Figure 7B). These experiments demonstrate that oscillatory transcription is inhibited 513 by the starvation-induced developmental diapause and that wild-type animals can re-initiate these 514 transcriptional patterns in a nutrient-dependent manner. 
BLMP-1 expression is actively regulated post-translationally by a conserved F-box protein,

516 DRE-1, that mediates the ubiquitination of target proteins which are degraded by the proteasome

517 (Horn et al., 2014). Given that blmp-1 is cyclically transcribed and its transcription is inhibited in

518 nutrient depleted conditions (Figure 7B), we hypothesized that the constitutive expression of

519 BLMP-1 during normal development may be altered during starvation. To test this, we repeated

520 the nutrition-mediated arrest experiments outlined above in strains harboring BLMP-1::GFP and

521 ELT-3::GFP translational fusions. The expression patterns of each translational fusion were

522 compared to a constitutively expressed monomeric GFP transgene (sur-5::GFP) to control for

523 potential changes in GFP half-life under starvation conditions. Surprisingly, BLMP-1::GFP

524 expression was diminished during starvation with $>50 \%$ of animals loosing detectible BLMP-

$5251::$ GFP expression after 12 hours of starvation. In contrast to the down-regulation of BLMP-

$526 \quad 1::$ GFP, most starved animals retained ELT-3::GFP and soluble GFP expression throughout the

527 starvation period with only a mild reduction after 24 hours (Figure 7C). As with the transcriptional

528 reporter for blmp-1 expression, BLMP-1::GFP expression was detectible again in most animals

529 within 2 hours or re-feeding and resumed normal, pre-starved expression levels after 5 hours

530 (Figure 7C). Similar changes in blmp-1 mRNA and BLMP-1 protein expression were seen in

531 freshly hatched and starved L1 (Figure S8). We conclude that BLMP-1 expression, both

532 transcriptionally and post translationally, is regulated by nutrient availability.

533 To examine if BLMP-1-dependent chromatin remodeling is also modulated by nutrient-

534 dependent signaling, we monitored compaction of lin-4 locus in animals that have entered the

535 acute starvation-dependent developmental diapause. At the mid-L3 stage (first VPC division), a

536 developmental time point that precedes lin-4 transcription during the L3 stage the lin-4 locus was

537 decompacted (Figure 7D). We then rapidly removed the bacteria food source to induce the L4-

538 specific developmental diapause. After 22 hours of starvation, $>90 \%$ of animals arrested at the

539 invagination stage of vulval development (L4.1-L4.2) which precedes fusion between the anchor

540 cell and vulval precursor cells on the ventral surface of the L4 larva (Mok et al., 2015). Analysis 
541 of lateral seam cell GFP-Lacl puffs sizes in starved animals demonstrated that the lin-4 loci in

542 seam cells were dramatically compacted when compared to the same loci in continuously

543 developing animals (Figure 7D). Re-plating of these starved animals lead to the rapid

544 decompaction of the lin-4 locus at a timepoint with BLMP-1 expression has resumed (Figure 7D).

545 Interestingly, the level of decompaction is greater in recovering animals that those found in non-

546 starved, continuously developing animals (Figure 7D). We also monitored lin-4::mCherry/LacO

547 loci in the seam cells of starved, L1-stage animals and also found that loci compacted in these

548 starvation conditions (Figure S8). In sum, these experiments indicate that dynamic chromatin

549 rearrangements are modulated by nutrient sensing.

550 Changes in BLMP-1 expression and the dramatic compaction and decompaction of

551 BLMP-1 target chromatin during starvation and recovery, respectively, suggests that BLMP-1

552 could play an essential role in modulating transcriptional output of critical developmental genes in

553 dynamic environments. To determine whether animals that lack blmp-1 and/or elt-3 can robustly

554 adapt to starvation, we repeated the food experiments as described in Figure 7A but maintained

555 the animals in starvation conditions for increasing periods of time. At the end of the starvation

556 period, animals were re-plated on normal food and monitored for developmental phenotypes by

557 scoring changes in col-19::GFP expression when the animals had reached adulthood (Figure 7E).

558 In these experiments, wild-type and elt-3(0) larvae re-initiate normal temporal patterning after 559 prolonged bouts of starvation (Figure 7E). In contrast, a minor fraction of blmp-1(0) mutants failed 560 to express col-19::GFP in hyp7 cells, a phenotype associated with weak reiterative heterochronic 561 mutants (Abbott et al., 2005; Hammell et al., 2009). The relatively mild blmp-1(0) phenotypes 562 were dramatically enhanced in blmp-1(0); elt-3(0) double mutants. These results indicate that

563 BLMP-1 and ELT-3 are essential for resuming normal temporal patterning after continuous 564 development has been interrupted by starvation. 


\section{Features of the oscillatory expression program impose constraints on modulating}

\section{8 transcriptional output}

569 Oscillatory patterns of gene expression are a ubiquitous feature of developmental systems where

570 they orchestrate repetitive biological functions and/or anticipate recurring environmental

571 conditions (Hasty et al., 2010). While most GRNs that program cyclical patterns of gene

572 expression utilize a basic negative feedback structure, specific features of their architecture

573 define the precision of the timekeeping mechanism and the ability to modulate and/or preserve

574 features of dynamic transcription. For instance, the periodicity of circadian gene expression

575 persists in the absence of external cues over a wide range of temperatures, yet changes in

576 lighting or nutrition can alter the phase of gene expression or the amplitude of clock target gene

577 transcription, respectively (Shigeyoshi et al., 1997; Trott and Menet, 2018). The genetic control

578 of plant root formation also employs a complex feedback loop that generates cyclical

579 transcriptional cycles that induce the formation of lateral roots at periodic positions along the

580 radial surfaces of the developing primary root. The transcriptional output of this GRN is

581 modulated by a hormone, auxin, that amplifies oscillatory transcription in a dose-dependent

582 manner (Moreno-Risueno et al., 2010). Exposure to light is required for maintaining the oscillatory

583 transcriptional patterns at the pre-branch sites (Kircher and Schopfer, 2016). Water availability

584 can also modulate the ability to the root clock to induce lateral root founder cell development.

585 Uneven water distribution on the surface of the developing primary root leads to a suppression

586 of LR development on the dryer root surface (Bao et al., 2014). Importantly, changes in water

587 distribution do not alter the cycling of core root clock components indicating the environment

588 can control clock output at the phenotypic level (Bao et al., 2014).

589 While the molecular components of the C. elegans expression oscillator are not fully

590 known, a detailed characterization of the systems-level behavior of this GRN indicates that two

591 features are hard-wired. First, the biological oscillator is directly tied to the molting cycle and 
592 gated by genetically-regulated developmental checkpoints (Hendriks et al., 2014; Schindler et

593 al., 2014). Under replete nutritional conditions, transcriptional cycling is continuous (Hendriks et

594 al., 2014). Removal of food either at hatching or just prior to the termination of the molt results

595 in a regulated arrest of the developmental clock at a precise point within the transcriptional cycle

596 (Figure 7B)(Hendriks et al., 2014). Re-initiation of the transcriptional clock (either from starved

597 L1-stage animals, dauer arrest, or the nutrition-mediated arrest points at each larval stage)

598 occurs at this same point (Hendriks et al. 2014). These features indicate that once initiated, the

599 transcriptional cycle is modular in nature and progresses until the next checkpoint. Secondly,

600 the periodicity of thousands of cyclically expressed transcripts scales inversely with temperature

601 over a wide dynamic range and the relative timing of expression for individual genes within the

602 transcriptional cycle is phase-locked (Hendriks et al., 2014; Kim et al., 2013).

603 Because the accordion-like, modular structure of this GRN constrains the relative timing

604 of transcriptional events within the cycle, other features of cyclically transcribed genes are likely

605 modulated independently of the clock. This adaption of transcriptional output to various

606 environments would be especially important for genes that are expressed at determinant phases

607 within the transcriptional cycle and function in dosage-sensitive manners. This feature is

608 exemplified by the heterochronic miRNAs that exhibit pulsatile transcriptional patterns at each

609 larval stage and also function to control discrete transitions of temporal cell fate at specific

610 developmental milestones (molting) (Ambros, 2011). It had been previously shown that LIN-42,

611 the C. elegans Period ortholog, functions to limit transcriptional output of this class of genes by

612 negatively regulating the overall duration of transcription within each larval stage (McCulloch and

613 Rougvie, 2014; Perales et al., 2014; Van Wynsberghe and Pasquinelli, 2014). In this manuscript,

614 we present evidence that BLMP-1 functions to antagonize LIN-42 activities by increasing the

615 duration of transcription for cyclically expressed genes. As with lin-42, blmp-1 is not essential

616 for the generation of cyclical expression patterns but plays a modulatory role in controlling the 
617 duration and amplitude of transcription (Figure 7G). How the interplay between these two

618 antagonistic modulators of gene expression achieves precise tuning of gene-dosage to confer

619 robustness and adaptivity to post-embryonic developmental programs is an exciting route for

620 further investigation given that BLMP-1 targets the lin-42 locus (Table S1).

622 BLMP-1 couples chromatin de-compaction and the regulation of transcriptional output

623 Here, we characterize a molecular role for BLMP-1 in regulating the transcriptional output of a

624 number of cyclically expressed genes through a mechanism involving the decompaction of

625 chromatin near its target gene loci. In contrast to the chromatin decompaction that occurs during

626 normal transcriptional activation (Dietzel et al., 2004; Tumbar et al., 1999; Yuzyuk et al., 2009),

627 we demonstrate that BLMP-1-dependent decompaction is temporally separated from the

628 activation of the target gene expression and can occur even when transcription is actively

629 repressed by other mechanisms (e.g. for the lin-4 gene during embryogenesis (Figure 6B and

630 D)(Ow et al., 2008). We hypothesize that this anticipatory priming mechanism constitutively

631 opens chromatin loci near BLMP-1 binding sites. These "primed" loci would then be accessible

632 to additional TFs whose binding capacity may be normally restricted by nucleosome complexes.

633 Once bound, these factors would induce the phased patterns of gene expression that define the

634 transcriptional cycle for each target gene (Figure $7 \mathrm{G}$ ). Because BLMP-1 binding sites are

635 associated with a multitude of genes that are expressed in diverse phases of the oscillatory

636 transcriptional pattern (Figure 3), we speculate that the BLMP-1-dependent "priming" activity is

637 not limited to a single partner TF but likely facilitates the association of several TFs that function

638 at distinct phases of the transcriptional cycle. In the absence of BLMP-1, many of these factors

639 would exhibit a reduced capacity to bind to their cognate binding sites. As a result, b/mp-1(0)

640 mutants exhibit broad changes in transcriptional dynamics. The diversity of these changes would

641 result in pleiotropic phenotypes whose only common feature would be a change in gene dosage 
642 across multiple GRNs. Notably, while our analysis focused on genes whose expression is

643 upregulated in the presence of BLMP-1, the mechanism we propose by which BLMP-1

644 modulates target transcription is equally capable of inhibiting transcription of target genes (by

645 increasing chromatin accessibility for transcriptional repressors).

646 The molecular mechanism that we propose for BLMP-1 in temporal gene regulation is

647 analogous to the role that the TF Zelda (ZLD) plays in establishing normal spatial gene regulation

648 during $D$. melanogaster embryogenesis. In this system, ZLD is ubiquitously expressed throughout

649 the embryo where it functions as a pioneer factor to rearrange chromatin near its binding sites

650 (Liang et al., 2008; Foo et al., 2014; McDaniel et al., 2019). Spatially ubiquitous ZLD activity

651 facilitates the transcriptional activation of genes that are co-targeted by the transcription factor

652 Dorsal (DL). Unlike ZLD, DL is expressed in a dorsal-ventral gradient that extends from the dorsal

653 surface of the embryo to the lateral edge. DL functions in a dosage-dependent manner to generate

654 a graded expression of target genes (Rushlow et al. 1989; Roth et al. 1989). Mutation of ZLD

655 binding sites in DL-dependent reporter transgenes results in the dramatic compression of reporter

656 gene expression along the dorsal-ventral axis (Yamada et al, 2019). Importantly, zld mutations

657 do not alter DL distribution along the dorsal-ventral axis suggesting that ZLD activity primes DL

658 targets by reorganizing chromatin architecture that would otherwise impair DL binding and

659 function. As with mutations of BLMP-1 in the context of temporal gene expression, zld mutations

660 additionally alter the duration of DL target expression (Yamada et al. 2019).

661

662 Regulation of BLMP-1 expression in response to nutrient availability coordinates

\section{3 transcriptional output}

664 We hypothesize that the re-initiation of transcription after starvation elicited by food is essential

665 for animals to re-format the chromatin landscape near their target genes after quiescence and

666 to rapidly modulate the transcriptional output of genes that control temporal patterning. In this 
667 manuscript we also show that blmp-1 expression is regulated by nutrient availability and is

668 important for the coordination of transcriptional output in a variety of conditions. Under 669 nutritionally replete growth conditions, blmp-1 transcription is both cyclical and auto-regulatory.

670 While blmp-1 expression at the mRNA level is highly dynamic, BLMP-1 protein expression is

671 highly stable and maintained at relatively constant levels during continuous growth (Hendriks et

672 al., 2014; Horn et al., 2014; Kim et al., 2013). Starvation elicits two main changes in blmp-1

673 expression that directly lead to a reduction in the priming activity. First, the cyclical transcription

674 of blmp-1 (as with most other cyclically expressed genes) is arrested when animals pause

675 development at a starvation-induced developmental checkpoint. Second, BLMP-1 expression is

676 rapidly curtailed during starvation. BLMP-1 expression is likely dampened by the combined

677 reduction in transcription and the constitutive activity of DRE-1 that functions independently of

678 starvation (Horn et al., 2014). This reduction of BLMP-1 expression is coincident with a re-

679 compaction of chromatin near BLMP-1 target genes. The establishment of non-permissive

680 chromatin architecture may be a common feature of quiescent cells as diverse cell types (from

681 yeast to human cells) exhibit these changes. It has also been previously suggested to control

682 inappropriate transcription in the context of development (Evertts et al., 2013; Laporte et al.,

6832016 Pinon, 1978; Rawlings et al., 2011; Rutledge et al., 2015; Swygert et al., 2019). The

684 regulation of BLMP-1 expression through nutrient availability is also important for the

685 coordination of gene expression after starvation as blmp-1(0) and blmp-1(0); elt-3(0) animals fail

686 to resume normal temporal patterning after starvation.

687 Finally, we hypothesize that the active control of BLMP-1 levels/activities may be

688 important for the adaption of global transcriptional patterns to the environment. The separate

689 genetic control of transcriptional timing and transcriptional output would enable gene expression

690 dynamics to be optimized to specific conditions and ensure developmental robustness.

691 Evidence that the levels of BLMP-1 protein are actively regulated are derived from the 
692 observation that dre-1(If) phenotypes result in an inappropriate over-expression of BLMP-1 and

693 a coincident increase in precocious developmental phenotypes (Horn et al., 2014). The active

694 control of BLMP-1 protein expression may play an important role in the adaptation of

695 transcriptional output to different temperatures where the periodicity of the transcriptional cycles

696 and developmental pace changes dramatically (Kim et al. 2013; Hendriks et al. 2014). This

697 homeostatic strategy, mediated by controlling the expression of a single pioneer transcription

698 factor, could buffer differences in transcriptional dynamics across the transcriptional cycle by

699 modulating chromatin accessibility.

700 


\section{EXPERIMENTAL METHODS}

702 See Star Materials and Methods Document.

703

704 ACKNOWLEDGEMENTS

705 We thank A. Zinovyeva, V. Ambros, L. M. Kutscher, and members of the Hammell laboratory for

706 critical review of this manuscript. We received C. elegans strains and recombinant DNAs from S.

707 Gasser, O. Hobert, L. Cochella. The ATIP/Avenir Young Investigator program of the CNRS

708 supported to W.K. W.K. initiated and performed part of this work while being a postdoctoral fellow

709 in the laboratories of Shai Shaham (S.S.) and Eric D. Siggia (E.D.S) at Rockefeller University,

710 supported by NIH grant R35NS105094 to S.S., NSF grant PHY 1502151 to E.D.S. and a

711 postdoctoral fellowship (LT000250/2013-C) from the Human Frontier Science Program (HFSP)

712 to W.K.. Cold Spring Harbor Laboratory, the Rita Allen Foundation, HIH NIGMS R01GM117406

713 supported C.M.H..

714

\section{AUTHOR CONTRIBUTIONS}

716 N.S., W.K., and C.M.H. designed, performed and analyzed most experiments and wrote the

717 manuscript. ChIP-seq experiments were performed by V.E. and S.E.. C.M.H and K.H-M. analyzed

718 sequencing data. Gel shift experiments were carried out by K.D.. Reporter construction and gene

719 expression analysis was carried out by C.M.H., N.S. and K.D.. Microfluidics experiments were

720 carried out by W.K.. Starvation experiments and LacO/Lacl experiments were carried out by N.S.

721 and C.M.H..

722

\section{DECLARATION OF INTERESTS}

724 The authors declare no competing interests 
Figure Legends:

727 Figure 1. blmp-1 and elt-3 antagonize lin-42 activity to control the expression of

728 heterochronic miRNAs. (A) Representative col-19::GFP expression patterns in wild-type, lin-

729 42(If), blmp-1(0); lin-42(If), and blmp-1(0); lin-42(If); elt-3(0) animals. (B) A quantification of the

730 col-19::GFP expression phenotypes of various mutant combinations in L4- and adult-staged

731 animals. (C) Expression patterns of BLMP-1::GFP and ELT-3::GFP transgenes in hypodermal

732 cells. $\mathrm{Sn}=$ Seam cell nuclei. (D) Representative images of mid-L4-staged wild-type or lin-42(If)

733 animals expressing a let-7::GFP-pest transcriptional reporter. S = seam cell. (E) Lateral seam cell

734 lineage of wild-type, blmp-1, and alg-1 mutants and experiments outlined in panel F. (F)

735 SCM::GFP is expressed in lateral seam cells and can be used to monitor cell division patterns

736 during larval development. Mid-L4-staged alg-1(0); blmp-1(RNAi) animals exhibit a

737 supernumerary number of SCM::GFP seam cells indicative of an inappropriate reiteration of seam

738 cell division programs. (G) Quantification of lateral seam cell numbers of mid-L4-staged animals

739 exposed to bacteria expressing dsRNAs complementary to elt-3, blmp-1 or both blmp-1 and elt-

740 3. Asterisks indicate statistically significant differences in phenotype $(p=<0.0001)$ calculated

741 from a two-tailed chi-square analysis.

743 Figure 2. blmp-1 and elt-3 regulate several additional morphological, behavioral, and

744 cellular phenotypes. (A) blmp-1(0); elt-3(0) animals exhibit a synthetic Dpy phenotype that

745 manifests during larval development. (B) Quantification of average animal length and width of

746 young adult animals of the indicated genotypes $(n=>20)$. Red bars indicate the mean and SEM.

747 (C) blmp-1(0) and blmp-1(0); elt-3(0) animals prematurely die after the L4-adult transition. (D) The

748 lethality of blmp-1(0); elt-3(0) adult animals correlates with an inability to shed the cuticle of a

749 supernumerary molt. (E) blmp-1(0); elt-3(0) inappropriately reanimate the expression of a mlt- 
751 transition or as gravid adults. (F) Major components of the vulval induction pathway (see text for

752 details). Components that are genetically involved in vulval induction are colored green whereas

753 those involved in Notch/lateral inhibition are colored black. The let-7 family miRNA miR-84 (red)

754 that regulates let-60 expression is temporally expressed in presumptive $2^{\circ}$ vulval cells. (G) blmp-

$7551(0)$ mutations enhance the expressivity and penetrance of let-60(n1046gf) muv phenotypes.

756 Animals of the indicated genotypes were scored for the number of vulval protrusions present on

757 the ventral side at the young adult stage $(n=>90)$. Brackets indicate statistically significant

758 differences in phenotype calculated from a two-tailed chi-square analysis. (H) Representative

759 images depicting Muv phenotypes associated with the indicated genotypes.

761 Figure 3. BLMP-1 and ELT-3 bind similar sites in the genome and are associated with the

762 putative regulatory regions of gens that exhibit cyclical expression patterns. (A) A matrix

763 of global pairwise factor co-association strengths as defined by promoter interval statistics

764 indicate that BLMP-1 and ELT-3 bind similar genomic regions. (B) The distribution of the peak

765 summit positions of BLMP-1 ChIP-seq data mapped relative to the TSS of protein coding genes

766 or to the +1 nucleotide of the DNA sequence encoding the miRNA precursor RNA. (C) A heat

767 map of ChIP-seq signals $+/-3 \mathrm{~kb}$ centered on the TSS of each of the 14,252 protein coding genes

768 measured in the developmental RNA-seq data set (Hendriks et al., 2014). Genes are organized

769 for each heat map in a 1:1 fashion according to their density of unique reads present in the BLMP-

7701 L1-stage ChIP-seq sample. (D) The C. elegans larval transcriptome can be categorized into two

771 dynamic expression classes (rising and oscillating) that are distinct from the predominantly flat

772 expression pattern of most mRNAs (grey region; 66.9\%) (Hendriks et al., 2014). (E) A graph

773 displaying the number of predicted rising or oscillating class targets for each of the 172 TFs

774 assayed by the ModEncode Projects (data derived from a total of 265 ChIP-seq data sets 
775 assayed). Red bars indicate the median (thick) with the interquartile range (thin). (F)

776 Quantification of GFP intensity for translational reporters of BLMP-1 and ELT-3 during the L4

777 stage. (G) A radar chart plotting oscillation amplitude over the phase of peak expression reveals

778 that BLMP-1 and ELT-3 bind the putative regulatory regions of periodically expressed genes in

779 all phases of the cyclical transcriptional cycle.

781 Figure 4. Conserved elements in the lin-4 promoter are both necessary and sufficient for

782 high-amplitude expression in hypodermal tissues. (A) A browser track overlaying the

783 predicted BLMP-1 consensus binding sites, the BLMP-1::GFP ChIP-seq signal from L1-staged

784 larvae, and conservation of these sequence features found in C. elegans, C. brenneri, C.

785 tropicalis, C. remanei, C. briggsae and C. japonica near the lin-4 locus and promoters used in the

786 expression analysis. Green bars indicate H3K4me3 ChIP-seq data tracks (indicative of active

787 promoters near transcriptional start sites). Black bars indicate the localization of open chromatin

788 as measured from ATAC-seq experiments (Daugherty et al., 2017). (B) Representative

789 measurements of full-length lin-4::GFP-pest expression levels in lateral seam cells. The

790 fluorescent intensity of five seam cells per animal were averaged and 8-15 animals were

791 measured per indicated L4 morphological substage. (C) Representative fluorescence and DIC

792 images of the full-length lin-4::GFP-pest transcriptional reporter in the hypodermal cells of

793 developing L4-staged animals. L4-stage numbers correspond to the defined sub-stages of vulval

794 morphological development $(\mathrm{S} n=$ seam cell nuclei, $\mathrm{h}=$ hyp7 cell nuclei, and $\mathrm{m}=$ muscle cell

795 nuclei) (Mok et al., 2015). (D) Measurements of seam cell expression levels for the various lin-

$7964:: G F P$-pest reporter constructs outlined in panel A at the L4.5 stage of development. $(n=5-10$

797 animals per transgenic strain) (E) Representative images of each transcriptional reporter outlined

798 in D. Individual labels defining cell types are the same as in panel C. (F) Position Weight Matrix

799 of the consensus BLMP-1 binding site calculated from ChIP-seq data outlined in Figure 3A and

800 two sequences located in the minCE element that conform to this consensus. (G) Gel shift 
801 experiments demonstrating that a GST fusion protein harboring the $5 \mathrm{ZnF}$ domains of BLMP-1

802 (but not GST alone) can bind to the two sequences outlined in panel F. (H) Comparison of the 803 GFP-pest expression patterns of gst-5::GFP-pest or (2xminCE)gst-5::GFP-pest in late L1-stage 804 animals. The pulsatile expression of the (2xminCE)gst-5::GFP-pest expression correlates with a 805 single pulse in the middle of each larval stage. For the developmental time course, a minimum of 806 forty animals expressing gst-5::GFP-pest or (2xminCE)gst-5::GFP-pest were scored.

807

808 Figure 5. blmp-1 modulates the amplitude and transcriptional duration of their cyclically

809 expressed targets. (A-C) Quantification of GFP-pest expression from the (2xminCE)gst-5,

810 C02E7.6 and moe-3 transcriptional reporters in L4-staged animals. Top panel for each reporter

811 indicates the average fluorescent intensity of GFP-pest expression in the lateral seam cells for

812 each morphologically defined L4 stage $((2 x \operatorname{minCE}) g s t-5=\mathrm{L} 4.5, \mathrm{C} 02 \mathrm{E} 7.6=\mathrm{L} 4.5$, and $\mathrm{moe}-3=$

813 L4.9) ( $n=7-14$ animals)( $\mathrm{Sn}=$ seam cell nuclei). (D) Estimation plot (Ho et al., 2019) showing the

814 duration of second larval stage (L2) for WT $(\mathrm{N}=92)$, blmp-1(0) $(\mathrm{N}=46)$, elt-3(0) $(\mathrm{N}=15)$ and blmp-

815 1(0); elt-3(0) animals $(\mathrm{N}=50)$. Upper row: Grey circles indicate L2 duration, measured by

816 observing cuticle shedding (molts) during long-term live imaging using a microfluidics device (Keil

817 et al., 2016). (see Methods). Black error bars indicate mean and standard deviation. Lower row:

818 Mean bootstrapped difference values compared to WT and 95\% confidence intervals of bootstrap

819 distributions (black error bars). (E) Merged DIC and fluorescence micrographs (after worm axis

820 straightening (Keil et al., 2016)) showing reporter expression at peak intensity in the L2 stage. (F)

821 ZK180.5::GFP-pest reporter intensity, averaged over the entire animal throughout development

822 as a function of time for WT (light red, $\mathrm{N}=23$, dark red average) and $b / m p-1(0)$ mutant (light blue,

$823 \mathrm{~N}=13$, dark blue average). Time courses are scaled such that molts (dashed grey lines) of

824 individual animals align with average WT molting timings. (G) Inset of (F), showing the

825 fluorescence intensity during the L2 stage of development. (H) L2 peak fluorescence intensity (left

826 most), onset phase of reporter expression (left middle), peak phase of reporter expression (right 
827 middle) and Output (AOC = area under the curve) (right most) for the ZK180.5::GFP-pest reporter

828 in WT (red circles, $\mathrm{N}=21$ ) and $b / m p-1(0)$ mutant (blue circles, $\mathrm{N}=13$ ). Black triangles indicate mean

829 bootstrapped difference values, blue error bars indicate 95\% confidence intervals of bootstrapped

830 difference distribution. (I,J) As (F, G) but for m/t-10::GFP-pest (WT, N=7; blmp-1(0), N=5).

831

832 Figure 6. The lin-4 locus is maintained in an open chromatin state in hypodermal cells in a

833 blmp-1-dependent manner. (A) Schematic of the transgenic array used for visualization of the

834 lin-4 locus in living animals. (B) Representative image of L1-staged animals expressing the GFP-

835 lacO-labeled lin-4 locus. (C) Analysis of the expression pattern of an endogenously GFP-tagged

836 translational fusion of BLMP-1 indicating that expression begins in hypodermal cells around the

837 bean stages and remains stably expressed throughout embryonic development. (D) Pictographs

838 depicting the compression level of the lin-4 locus/GFP-Lacl foci in differentially staged wild-type

839 and blmp-1(0) mutant embryos. Yellow arrows indicate the location of hypodermal nuclei. (E)

840 Quantification of the percentage of wild-type or blmp-1(0) late-stage (pretzel) embryos that exhibit

841 decompacted lin-4 loci in hypodermal cells. (F) Close-up images of representative seam cell and

842 neuronal nuclei from L3-staged wild-type and blmp-1(0) mutant larva. GFP images are the

843 maximum intensity projections obtained for each relevant nuclei. (G) Quantification of the sizes

844 of the nuclear GFP-Lacl foci in various cell types of wild-type and b/mp-1(0) mutant animals.

845 Boxes and median line indicate the interquartile range. Whiskers cover 10-90th percentile. Circles

846 represent outliers. Brackets indicate statistically significant differences in "puffs size" calculated

847 from a two-tailed chi-square analysis.

848

849 Figure 7. Dynamic gene expression is halted during nutrition-mediated developmental 850 arrest and BLMP-1 and ELT-3 are essential for the recovery of normal temporal patterning

851 after starvation. (A) A schematic of the starvation experiment used to measure nutrient852 depended changes in the expression patterns of cyclically expressed genes. (B) Continuous 
853 cycling of gene expression is arrested during starvation conditions and is reinitiated in a

854 coordinated manner when animals resume development. For each timepoint, 70-200 animals

855 were scored. (C) BLMP-1::GFP expression is down-regulated during starvation conditions and

856 expression is rapidly resumed when animals reinitiate development. (D) The lin-4 locus is

857 compacted during starvation conditions in lateral seam cells. (E) A schematic of the starvation-

858 mediated arrest and re-feeding experiment used to measure the ability of animals to resume

859 normal temporal patterning after starvation. (F) The resumption of normal temporal patterning

860 after starvation requires blmp-1 and elt-3 activity. Defects in temporal development were

861 monitored by measuring the col-19::GFP expression phenotypes in re-fed animals after they

862 resumed growth for 24 hours after the indicated period of starvation. ( $n=>100$ per condition) (G)

863 Representative pictographs of the col-19::GFP phenotypes in wild-type and blmp-1(0); elt-3(0)

864 mutants after animals have recovered from 5 days of starvation. $(\mathbf{H})$ A diagram outlining the

865 expression features of a single transcriptional cycle in wild-type and $b / m p-1(0)$ animals. (I) A

866 model for how BLMP-1 and ELT-3 function to regulate transcriptional output during larval

867 development. (J) A diagram outlining the changes of blmp-1 mRNA and BLMP-1 protein levels

868 during normal grown and during starvation conditions. 
871 Supplemental Figure 1. blmp-1(0) mutations enhance the heterochronic phenotypes other

872 reiterative mutants. (A) The normal expression pattern of a col-19::GFP transcriptional reporter

873 is altered in various, weak reiterative heterochronic mutants. This transcriptional reporter is

874 usually expressed in both the lateral seam and hyp7 cells of the skin. While most mutant animals

875 exhibit the wild-type expression pattern, combining these mutations with blmp-1(0) dramatically

876 alters the expression patterns. A majority of double mutant animals only express col-19::GFP in

877 the lateral seam cells. In addition, most double mutant animals inappropriately reiterate L4 seam

878 cell division patterns resulting in supernumerary seam cells as adults. (B) Quantification of the

879 col-19::GFP expression phenotypes in indicated mutant animals.

880

881 Supplemental Figure 2. BLMP-1 and ELT-3 binding sites are enriched in the promoters of

882 cyclically transcribed genes and are near sites of dynamically open chromatin. Browser

883 tracks overlaying the BLMP-1::GFP and ELT-3::GFP ChIP-seq signal from L1-staged larvae for

884 the indicated genomic loci. Green bars indicate H3K4me3 ChIP-seq data tracks (indicative of

885 active promoters near transcriptional start sites). Black bars indicate the localization of open

886 chromatin as measured from ATAC-seq experiments in extracts derived from embryonic, L3-

887 staged or adult animals (Daugherty et al., 2017).

889 Supplemental Figure 3. BLMP-1 binding sites are enriched in the cyclically expressed 890 classes of transcripts identified in the Kim et al. high-resolution transcriptome data. (A)

891 Clusters of genes with cycling or graded expression patters as defined by Kim et al. 2013 (Kim et 892 al., 2013). Graphs for each cluster illustrate the average, normalized expression profiles. dashed 893 grey lines indicate transitional between larval stages at $20^{\circ} \mathrm{C}$. The cluster number is indicated in 894 the upper left corner of each graph. (B) A graph illustrating the distribution of genes in each 
895 expression cluster. Pie chart illustrates the proportion of genes (10.1\%) from the transcriptomic

896 data that produce mRNA that are in cycling clusters one through six. (C) The predicted enrichment

897 of BLMP-1 targets in each of the twelve expression clusters defined in the Kim et al. 2013 mRNA-

898 seq analysis. Error bars are based on random counting statistics. $P$ values were computed by

899 Fisher's exact test. Data are shown for all clusters individually. ${ }^{* *}$ indicates a $\mathrm{p}$ values $<10^{-2}$ and

$900^{* * * *}$ indicates a $\mathrm{p}$ value $<10^{-4}$. All calculations can be found in Table S3.

901

902 Supplemental Figure 4. Cyclical expression of the (2xminCE)gst-5::GFP-pest transgene is

903 dependent on blmp-1 activity. (A) Pictographs exhibiting expression of gst-5::GFP-pest and

904 (2xminCE)gst-5::GFP-pest reporters in wild type and blmp-1(0) mutant animals in the mid L4

905 stage of development. (B) Quantification of individual expression levels of for the gst-5::GFP-pest

906 and (2xminCE)gst-5::GFP-pest reporters. $\mathrm{n}=>18$ for each reporter and or genetic context.

907 Expression of the gst-5::GFP-pest reporter was not altered in blmp-1(0) animals (data not

908 shown).

909

910 Supplemental Figure 5. Duration times of larval stages are similar in wild-type, blmp-1(0),

911 elt-3(0) and blmp-1(0); elt-3(0) mutant backgrounds. (A) Estimation plot (Ho et al., 2019)

912 showing the duration of second larval stage (L3) for WT $(\mathrm{N}=81)$, blmp-1(0) $(\mathrm{N}=36)$, elt-3(0) $(\mathrm{N}=8)$

913 and blmp-1(0); elt-3(0) animals $(\mathrm{N}=40)$. (B) Estimation plot showing the duration of second larval

914 stage (L4) for WT $(\mathrm{N}=51)$, blmp-1(0) $(\mathrm{N}=22)$, elt-3(0) $(\mathrm{N}=6)$ and blmp-1(0); elt-3(0) animals $915(\mathrm{~N}=30)$.

916

917 Supplemental Figure 6. elt-3(0) mutations only minimally enhance expression defects of 918 ZK180.5::GFP-pest and mIt-10::GFP-pest reporters in blmp-1(0) mutants. (A) A browser 919 track overlaying the BLMP-1::GFP and ELT-3::GFP ChIP-seq signal near the ZK180.5 locus. 920 Green bars indicate H3K4me3 ChIP-seq data tracks (indicative of active promoters near 
921 transcriptional start sites). Black bars indicate the localization of open chromatin as measured

922 from ATAC-seq experiments in extracts derived from embryonic, L3-staged or adult animals

923 (Daugherty et al., 2017). (B) A comparison the L2-L3 staged traces of ZK180.5::GFP-pest

924 expression and quantification of the features of these patterns in wild-type animals and $b / \mathrm{mp}-1(0)$

925 mutants. (C) Same as in B except for wild-type and blmp-1(0); elt-3(0) mutants. (D) Browser track

926 for the mlt-10 locus illustrating the same genomic features as in panel A. (E) A comparison the

927 L2-L3 staged traces of mlt-10::GFP-pest expression and quantification of the features of these

928 patterns in wild-type animals and blmp-1(0) mutants. (F) A comparison the L2-L3 staged traces

929 of $m / t-10:: G F P$-pest expression and quantification of the features of these patterns in wild-type

930 animals and elt-3(0) mutants. (G) A comparison the L2-L3 staged traces of m/t-10::GFP-pest

931 expression and quantification of the features of these patterns in wild-type animals and b/mp-1(0);

932 elt-3(0) mutants.

933

934 Supplemental Figure 7. blmp-1 expression is auto-regulatory. (A) Browser track of the blmp-1

935 genomic region depicting the enrichment of BLMP-1 binding sites in the promoter of the blmp-1

936 as defined by BLMP-1::GFP ChIP-seq signal in each stage of larval development. (B) A

937 transcriptional reporter harboring a $6.2 \mathrm{~kb}$ the upstream regulatory sequences from the $b / \mathrm{mp}-1$

938 gene was fused to a GFP-pest reporter. Wild-type and blmp-1(0) animals expressing an

939 extrachromsomal version of this transgene were stage as L1s and then plated onto normal NGM

940 plates containing OP50. To measure the temporal expression patterns, $>50$ animals were scored

941 for GFP expression every 2 hours. In wild-type animals, this reporter is expressed in a cyclical

942 manner consistent with characterization of the endogenous b/mp-1 transcripts (Hendriks et al.,

9432014 ; Kim et al., 2013). In contrast, a majority of blmp-1(0) animals fail to express detectible GFP-

944 pest expression in parallel cultures.

945 
946 Supplemental Figure 8. BLMP-1 expression and priming activities are repressed during L1-

947 stage arrests. (A) BLMP-1 expression is present in developing embryos, reduced in arrested L1

948 animals and re-expressed in animals that have been re-fed. (B) Quantification of the number of

949 puffed lin-4::mCherry-pest/LacO loci as a percentage of animals with greater than 3 puffs in the

950 seam cells of one lateral side of animals in the indicated conditions.

951

952 Supplemental Tables:

953 Supplemental Table 1. Summary of ChIP-seq experiments for BLMP-1::GFP and ELT-

954 3::GFP. Tables include lists of calculated peaks that were assigned to protein coding and non-

955 coding genes for each of the 5 ChIP-seq data sets presented in Figure 3. Includes peak location,

956 distance to assigned gene, gene name, and amplitude and phase information (if applicable).

957 Table also includes various comparisons of ChIP-seq data sets for co-targeting.

958

959 Supplemental Table 2. Summary of the analysis of ModEncode ChIP-seq data sets. Includes

960 the gene class assignments (flat, oscillating, rising; as defined by Hendriks et al.) for each TF

961 profiled by the ModEncode project (Hendriks et al., 2014).

962

963 Supplemental Table 3. Comparison of the enrichment of predicted BLMP-1 targets in the 964 various expression clusters as defined by Kim et al. 2013 (Kim et al., 2013). 
966 Bibliography

967 Abbott, A.L., Alvarez-Saavedra, E., Miska, E.A., Lau, N.C., Bartel, D.P., Horvitz, H.R., and 968 Ambros, V. (2005). The let-7 MicroRNA family members mir-48, mir-84, and mir-241 function 969 together to regulate developmental timing in Caenorhabditis elegans. Developmental cell 9, 970 403-414.

Abrahante, J.E., Miller, E.A., and Rougvie, A.E. (1998). Identification of heterochronic mutants in Caenorhabditis elegans. Temporal misexpression of a collagen::green fluorescent protein fusion gene. Genetics 149, 1335-1351.

Ambros, V. (2011). MicroRNAs and developmental timing. Current opinion in genetics \&amp; development 21, 511-517.

Ambros, V., and Horvitz, H.R. (1984). Heterochronic mutants of the nematode Caenorhabditis elegans. Science 226, 409-416.

Angeles-Albores, D., N Lee, R.Y., Chan, J., and Sternberg, P.W. (2016). Tissue enrichment analysis for $\mathrm{C}$. elegans genomics. BMC bioinformatics 17, 366-310.

Araya, C.L., Kawli, T., Kundaje, A., Jiang, L., Wu, B., Vafeados, D., Terrell, R., Weissdepp, P., Gevirtzman, L., Mace, D., et al. (2014). Regulatory analysis of the C. elegans genome with spatiotemporal resolution. Nature 512, 400-405.

990

Bao, Y., Aggarwal, P., Robbins, N.E., 2nd, Sturrock, C.J., Thompson, M.C., Tan, H.Q., Tham, C., Duan, L., Rodriguez, P.L., Vernoux, T., et al. (2014). Plant roots use a patterning mechanism to position lateral root branches toward available water. Proc Natl Acad Sci U S A 111, 93199324.

Beitel, G.J., Clark, S.G., and Horvitz, H.R. (1990). Caenorhabditis elegans ras gene let-60 acts as a switch in the pathway of vulval induction. Nature 348, 503-509.

Bracht, J.R., Van Wynsberghe, P.M., Mondol, V., and Pasquinelli, A.E. (2010). Regulation of lin4 miRNA expression, organismal growth and development by a conserved RNA binding protein 1000 Furlan, S.N., Steemers, F.J., et al. (2017). Comprehensive single-cell transcriptional profiling of a multicellular organism. Science 357, 661-667.

Cochella, L., and Hobert, O. (2012). Embryonic priming of a miRNA locus predetermines postmitotic neuronal left/right asymmetry in C. elegans. Cell 151, 1229-1242.

1011

Daugherty, A.C., Yeo, R.W., Buenrostro, J.D., Greenleaf, W.J., Kundaje, A., and Brunet, A. (2017). Chromatin accessibility dynamics reveal novel functional enhancers in C. elegans. Genome research 27, 2096-2107. 
1012

1013

1014

1015

1016

1017

1018

1019

1020

1021

1022

1023

1024

1025

1026

1027

1028

1029

1030

1031

1032

1033

1034

1035

1036

1037

1038

1039

1040

1041

1042

1043

1044

1045

1046

1047

1048

1049

1050

1051

1052

1053

1054

1055

1056

1057

1058

1059

1060

Dietzel, S., Zolghadr, K., Hepperger, C., and Belmont, A.S. (2004). Differential large-scale chromatin compaction and intranuclear positioning of transcribed versus non-transcribed transgene arrays containing beta-globin regulatory sequences. J Cell Sci 117, 4603-4614.

Dufourt, J., Trullo, A., Hunter, J., Fernandez, C., Lazaro, J., Dejean, M., Morales, L., Nait-Amer, S., Schulz, K.N., Harrison, M.M., et al. (2019). Publisher Correction: Temporal control of gene expression by the pioneer factor Zelda through transient interactions in hubs. Nat Commun 10, 315.

Edelman, T.L., McCulloch, K.A., Barr, A., Frokjaer-Jensen, C., Jorgensen, E.M., and Rougvie, A.E. (2016). Analysis of a lin-42/period Null Allele Implicates All Three Isoforms in Regulation of Caenorhabditis elegans Molting and Developmental Timing. G3 (Bethesda) 6, 4077-4086.

Evertts, A.G., Manning, A.L., Wang, X., Dyson, N.J., Garcia, B.A., and Coller, H.A. (2013). H4K20 methylation regulates quiescence and chromatin compaction. Mol Biol Cell 24, 30253037.

Fakhouri, T.H., Stevenson, J., Chisholm, A.D., and Mango, S.E. (2010). Dynamic chromatin organization during foregut development mediated by the organ selector gene PHA-4/FoxA. PLoS Genet 6.

Feinbaum, R., and Ambros, V. (1999). The timing of lin-4 RNA accumulation controls the timing of postembryonic developmental events in Caenorhabditis elegans. Developmental biology 210, 87-95.

Fong, H.T., Hagen, T., and Inoue, T. (2020). LDB1 and the SWI/SNF complex participate in both transcriptional activation and repression by Caenorhabditis elegans BLIMP1/PRDM1. Biochim Biophys Acta Gene Regul Mech 1863, 194577.

Foo, S.M., Sun, Y., Lim, B., Ziukaite, R., O'Brien, K., Nien, C.Y., Kirov, N., Shvartsman, S.Y., and Rushlow, C.A. (2014). Zelda potentiates morphogen activity by increasing chromatin accessibility. Curr Biol 24, 1341-1346.

Frand, A.R., Russel, S., and Ruvkun, G. (2005). Functional genomic analysis of C. elegans molting. PLoS biology 3 , e312.

Frezal, L., and Felix, M.A. (2015). C. elegans outside the Petri dish. Elife 4.

Garcia-Bellido, A. (1975). Genetic control of wing disc development in Drosophila. Ciba Found Symp 0, 161-182.

Hammell, C.M., Lubin, I., Boag, P.R., Blackwell, T.K., and Ambros, V. (2009). nhl-2 Modulates microRNA activity in Caenorhabditis elegans. Cell 136, 926-938.

Han, M., and Sternberg, P.W. (1990). let-60, a gene that specifies cell fates during C. elegans vulval induction, encodes a ras protein. Cell 63, 921-931.

Hasty, J., Hoffmann, A., and Golden, S. (2010). Systems biology of cellular rhythms: from cacophony to symphony. Curr Opin Genet Dev 20, 571-573. 
Hayes, G.D., Frand, A.R., and Ruvkun, G. (2006). The mir-84 and let-7 paralogous microRNA genes of Caenorhabditis elegans direct the cessation of molting via the conserved nuclear hormone receptors NHR-23 and NHR-25. Development 133, 4631-4641.

Hedgecock, E.M., and White, J.G. (1985). Polyploid tissues in the nematode Caenorhabditis elegans. Dev Biol 107, 128-133.

Hendriks, G.-J., Gaidatzis, D., Aeschimann, F., and Großhans, H. (2014). Extensive Oscillatory Gene Expression during C. elegans Larval Development. Mol Cell 53, 380-392.

Ho, J., Tumkaya, T., Aryal, S., Choi, H., and Claridge-Chang, A. (2019). Moving beyond P values: data analysis with estimation graphics. Nat Methods 16, 565-566.

Horn, M., Geisen, C., Cermak, L., Becker, B., Nakamura, S., Klein, C., Pagano, M., and Antebi, A. (2014). DRE-1/FBXO11-Dependent Degradation of BLMP-1/BLIMP-1 Governs C. elegans Developmental Timing and Maturation. Developmental cell.

Hsu, H.-T., Chen, H.-M., Yang, Z., Wang, J., Lee, N.K., Burger, A., Zaret, K., Liu, T., Levine, E., and Mango, S.E. (2015). TRANSCRIPTION. Recruitment of RNA polymerase II by the pioneer transcription factor PHA-4. Science 348, 1372-1376.

Hurley, J.M., Loros, J.J., and Dunlap, J.C. (2016). Circadian Oscillators: Around the Transcription-Translation Feedback Loop and on to Output. Trends Biochem Sci 41, 834-846.

Janes, J., Dong, Y., Schoof, M., Serizay, J., Appert, A., Cerrato, C., Woodbury, C., Chen, R., Gemma, C., Huang, N., et al. (2018). Chromatin accessibility dynamics across C. elegans development and ageing. Elife 7.

Jeon, M., Gardner, H.F., Miller, E.A., Deshler, J., and Rougvie, A.E. (1999). Similarity of the C. elegans developmental timing protein LIN-42 to circadian rhythm proteins. Science 286, 11411146.

Kanodia, J.S., Rikhy, R., Kim, Y., Lund, V.K., DeLotto, R., Lippincott-Schwartz, J., and Shvartsman, S.Y. (2009). Dynamics of the Dorsal morphogen gradient. Proc Natl Acad Sci U S A $106,21707-21712$.

Keil, W., Kutscher, L.M., Shaham, S., and Siggia, E.D. (2016). Long-Term High-Resolution Imaging of Developing C. elegans Larvae with Microfluidics. Developmental cell.

Kim, D.h., Grün, D., and van Oudenaarden, A. (2013). Dampening of expression oscillations by synchronous regulation of a microRNA and its target. Nat Genet 45, 1337-1344.

Kircher, S., and Schopfer, P. (2016). Priming and positioning of lateral roots in Arabidopsis. An approach for an integrating concept. J Exp Bot 67, 1411-1420.

Laporte, D., Courtout, F., Tollis, S., and Sagot, I. (2016). Quiescent Saccharomyces cerevisiae forms telomere hyperclusters at the nuclear membrane vicinity through a multifaceted 
1109

1110

1111

1112

1113

1114

1115

1116

1117

1118

1119

1120

1121

1122

1123

1124

1125

1126

1127

1128

1129

1130

1131

1132

1133

1134

1135

1136

1137

1138

1139

1140

1141

1142

1143

1144

1145

1146

1147

1148

1149

1150

1151

1152

1153

1154

1155

1156 mechanism involving Esc1, the Sir complex, and chromatin condensation. Mol Biol Cell 27, 1875-1884.

Lee, R.C., Feinbaum, R.L., and Ambros, V. (1993). The C. elegans heterochronic gene lin-4 encodes small RNAs with antisense complementarity to lin-14. Cell 75, 843-854.

Li, M., Jones-Rhoades, M.W., Lau, N.C., Bartel, D.P., and Rougvie, A.E. (2005). Regulatory mutations of mir-48, a C. elegans let-7 family MicroRNA, cause developmental timing defects. Developmental cell 9, 415-422.

Liang, G., Lin, J.C., Wei, V., Yoo, C., Cheng, J.C., Nguyen, C.T., Weisenberger, D.J., Egger, G., Takai, D., Gonzales, F.A., et al. (2004). Distinct localization of histone H3 acetylation and H3-K4 methylation to the transcription start sites in the human genome. Proc Natl Acad Sci U S A $101,7357-7362$.

Liang, H.L., Nien, C.Y., Liu, H.Y., Metzstein, M.M., Kirov, N., and Rushlow, C. (2008). The zincfinger protein Zelda is a key activator of the early zygotic genome in Drosophila. Nature 456, 400-403.

Mann, R.S., and Carroll, S.B. (2002). Molecular mechanisms of selector gene function and evolution. Curr Opin Genet Dev 12, 592-600.

McCulloch, K.A., and Rougvie, A.E. (2014). Caenorhabditis elegans period homolog lin-42 regulates the timing of heterochronic miRNA expression. Proceedings of the National Academy of Sciences of the United States of America 111, 15450-15455.

McDaniel, S.L., Gibson, T.J., Schulz, K.N., Fernandez Garcia, M., Nevil, M., Jain, S.U., Lewis, P.W., Zaret, K.S., and Harrison, M.M. (2019). Continued Activity of the Pioneer Factor Zelda Is Required to Drive Zygotic Genome Activation. Mol Cell 74, 185-195 e184.

Meister, P., Towbin, B.D., Pike, B.L., Ponti, A., and Gasser, S.M. (2010). The spatial dynamics of tissue-specific promoters during C. elegans development. Genes \&amp; development 24, 766-782.

Mello, C.C., Kramer, J.M., Stinchcomb, D., and Ambros, V. (1991). Efficient gene transfer in C.elegans: extrachromosomal maintenance and integration of transforming sequences. The EMBO journal 10, 3959-3970.

Mok, D.Z.L., Sternberg, P.W., and Inoue, T. (2015). Morphologically defined sub-stages of C. elegans vulval development in the fourth larval stage. BMC developmental biology 15, 26.

Monsalve, G.C., Van Buskirk, C., and Frand, A.R. (2011). LIN-42/PERIOD controls cyclical and developmental progression of C. elegans molts. Current biology : CB 21, 2033-2045.

Moreno-Risueno, M.A., Van Norman, J.M., Moreno, A., Zhang, J., Ahnert, S.E., and Benfey, P.N. (2010). Oscillating gene expression determines competence for periodic Arabidopsis root branching. Science 329, 1306-1311. 
Mutlu, B., Chen, H.M., Gutnik, S., Hall, D.H., Keppler-Ross, S., and Mango, S.E. (2019). Distinct functions and temporal regulation of methylated histone $\mathrm{H} 3$ during early embryogenesis. Development 146.

Mutlu, B., Chen, H.M., Moresco, J.J., Orelo, B.D., Yang, B., Gaspar, J.M., Keppler-Ross, S., Yates, J.R., 3rd, Hall, D.H., Maine, E.M., et al. (2018). Regulated nuclear accumulation of a histone methyltransferase times the onset of heterochromatin formation in C. elegans embryos. Sci Adv 4, eaat6224.

Niu, W., Lu, Z.J., Zhong, M., Sarov, M., Murray, J.I., Brdlik, C.M., Janette, J., Chen, C., Alves, P., Preston, E., et al. (2011). Diverse transcription factor binding features revealed by genomewide ChIP-seq in C. elegans. Genome research 21, 245-254.

Ow, M.C., Martinez, N.J., Olsen, P.H., Silverman, H.S., Barrasa, M.I., Conradt, B., Walhout, A.J., and Ambros, V. (2008). The FLYWCH transcription factors FLH-1, FLH-2, and FLH-3 repress embryonic expression of microRNA genes in C. elegans. Genes \&amp; development $22,2520-2534$.

Patel, T., and Hobert, O. (2017). Coordinated control of terminal differentiation and restriction of cellular plasticity. eLife 6, 249.

Perales, R., King, D.M., Aguirre-Chen, C., and Hammell, C.M. (2014). LIN-42, the Caenorhabditis elegans PERIOD homolog, Negatively Regulates MicroRNA Transcription. PLoS genetics 10, e1004486.

Pinon, R. (1978). Folded chromosomes in non-cycling yeast cells: evidence for a characteristic g0 form. Chromosoma 67, 263-274.

Rawlings, J.S., Gatzka, M., Thomas, P.G., and Ihle, J.N. (2011). Chromatin condensation via the condensin II complex is required for peripheral T-cell quiescence. EMBO J 30, 263-276.

Roth, S., Stein, D., and Nusslein-Volhard, C. (1989). A gradient of nuclear localization of the dorsal protein determines dorsoventral pattern in the Drosophila embryo. Cell 59, 1189-1202.

Rougvie, A.E., and Moss, E.G. (2013). Developmental Transitions in C. elegans Larval Stages. Curr Top Dev Biol 105, 153-180.

Rushlow, C.A., Han, K., Manley, J.L., and Levine, M. (1989). The graded distribution of the dorsal morphogen is initiated by selective nuclear transport in Drosophila. Cell 59, 1165-1177. undergoes significant topological reorganization in quiescence. Nucleic Acids Res 43, 82998313.

Schindler, A.J., Baugh, L.R., and Sherwood, D.R. (2014). Identification of late larval stage developmental checkpoints in Caenorhabditis elegans regulated by insulin/IGF and steroid hormone signaling pathways. PLoS genetics 10, e1004426. 
Schulz, K.N., Bondra, E.R., Moshe, A., Villalta, J.E., Lieb, J.D., Kaplan, T., McKay, D.J., and Harrison, M.M. (2015). Zelda is differentially required for chromatin accessibility, transcription factor binding, and gene expression in the early Drosophila embryo. Genome Res 25, 17151726.

Shigeyoshi, Y., Taguchi, K., Yamamoto, S., Takekida, S., Yan, L., Tei, H., Moriya, T., Shibata, S., Loros, J.J., Dunlap, J.C., et al. (1997). Light-induced resetting of a mammalian circadian

Stathopoulos, A., and Levine, M. (2002). Dorsal gradient networks in the Drosophila embryo. Dev Biol 246, 57-67.

Sulston, J.E., and Horvitz, H.R. (1977). Post-embryonic cell lineages of the nematode, Caenorhabditis elegans. Developmental biology 56, 110-156.

Sun, Y., Nien, C.Y., Chen, K., Liu, H.Y., Johnston, J., Zeitlinger, J., and Rushlow, C. (2015). Zelda overcomes the high intrinsic nucleosome barrier at enhancers during Drosophila zygotic genome activation. Genome Res 25, 1703-1714.

Swygert, S.G., Kim, S., Wu, X., Fu, T., Hsieh, T.H., Rando, O.J., Eisenman, R.N., Shendure, J., McKnight, J.N., and Tsukiyama, T. (2019). Condensin-Dependent Chromatin Compaction Represses Transcription Globally during Quiescence. Mol Cell 73, 533-546 e534.

Tennessen, J.M., Gardner, H.F., Volk, M.L., and Rougvie, A.E. (2006). Novel heterochronic functions of the Caenorhabditis elegans period-related protein LIN-42. Developmental biology 289, 30-43.

Tennessen, J.M., Opperman, K.J., and Rougvie, A.E. (2010). The C. elegans developmental timing protein LIN-42 regulates diapause in response to environmental cues. Development 137, 3501-3511.

Towbin, B.D., Gonzalez-Aguilera, C., Sack, R., Gaidatzis, D., Kalck, V., Meister, P., Askjaer, P., and Gasser, S.M. (2012). Step-wise methylation of histone H3K9 positions heterochromatin at the nuclear periphery. Cell 150, 934-947.

Trott, A.J., and Menet, J.S. (2018). Regulation of circadian clock transcriptional output by CLOCK:BMAL1. PLoS Genet 14, e1007156.

Tuck, S. (2014). The control of cell growth and body size in Caenorhabditis elegans. Experimental cell research 321, 71-76.

Tumbar, T., Sudlow, G., and Belmont, A.S. (1999). Large-scale chromatin unfolding and remodeling induced by VP16 acidic activation domain. J Cell Biol 145, 1341-1354.

Van Wynsberghe, P.M., Kai, Z.S., Massirer, K.B., Burton, V.H., Yeo, G.W., and Pasquinelli, A.E. (2011). LIN-28 co-transcriptionally binds primary let-7 to regulate miRNA maturation in Caenorhabditis elegans. Nat Struct Mol Biol 18, 302-308. 
1253 Van Wynsberghe, P.M., and Pasquinelli, A.E. (2014). Period homolog LIN-42 regulates miRNA 1254 transcription to impact developmental timing. Worm 3, e974453.

Xu, Z., Chen, H., Ling, J., Yu, D., Struffi, P., and Small, S. (2014). Impacts of the ubiquitous factor Zelda on Bicoid-dependent DNA binding and transcription in Drosophila. Genes Dev 28, 608-621.

Yamada, S., Whitney, P.H., Huang, S.K., Eck, E.C., Garcia, H.G., and Rushlow, C.A. (2019). The Drosophila Pioneer Factor Zelda Modulates the Nuclear Microenvironment of a Dorsal Target Enhancer to Potentiate Transcriptional Output. Curr Biol 29, 1387-1393 e1385. mes-2/E(z) promotes the transition from developmental plasticity to differentiation in $C$. elegans embryos. Dev Cell 16, 699-710. 
A

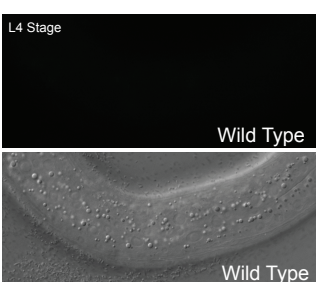

Wild Type

B

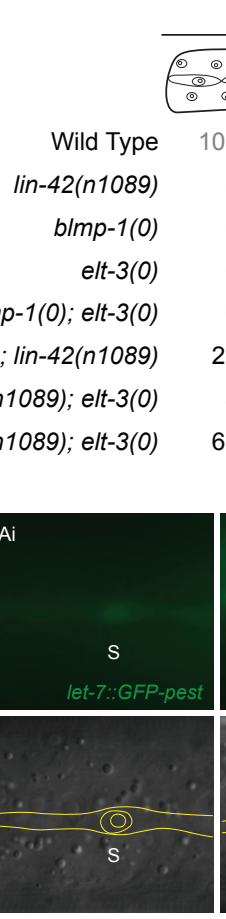

E

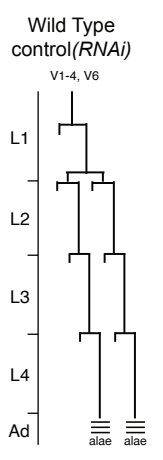

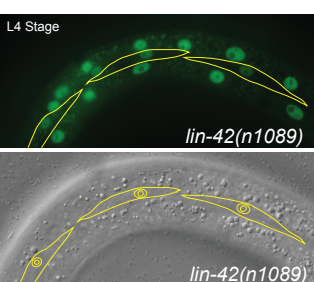
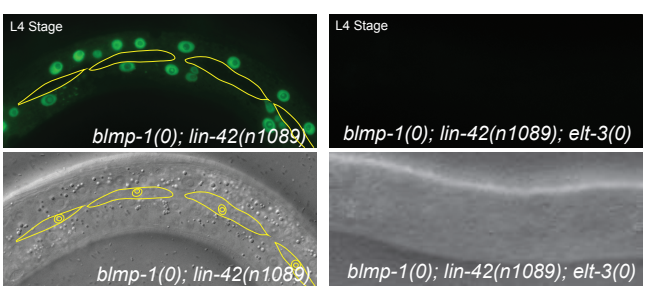

L4 Stage
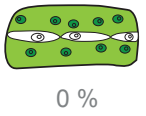

$0 \%$

$0 \%$

$0 \%$

$0 \%$

$72 \%$

$21 \%$

$35 \%$

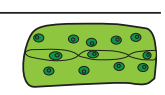

$0 \% 35$

$92 \% \quad 25$

$0 \% \quad 35$

$0 \% \quad 35$

$0 \% \quad 39$

$0 \% \quad 25$

$71 \% \quad 28$

$0 \% \quad 34$

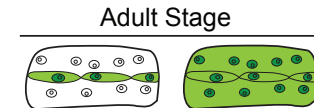

$0 \%$

$0 \%$

$1 \%$

$0 \%$

$5 \%$

$0 \%$

$0 \%$

$0 \%$
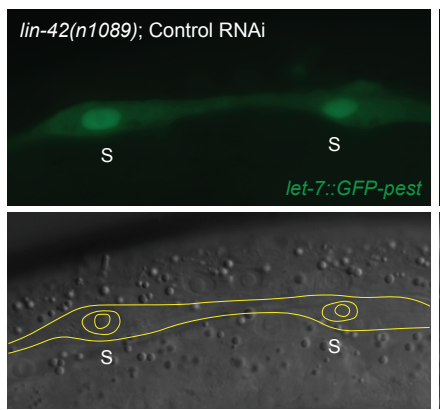

F

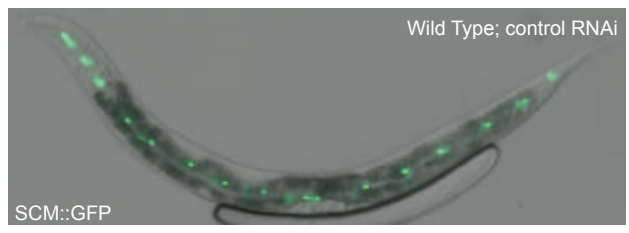

alg-1(0); blmp-1 RNAi

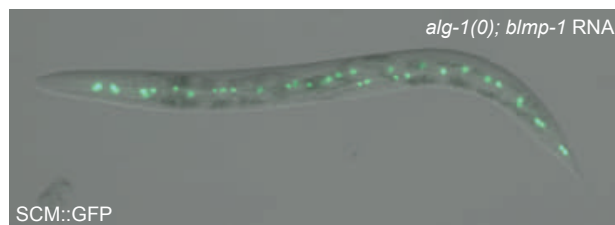

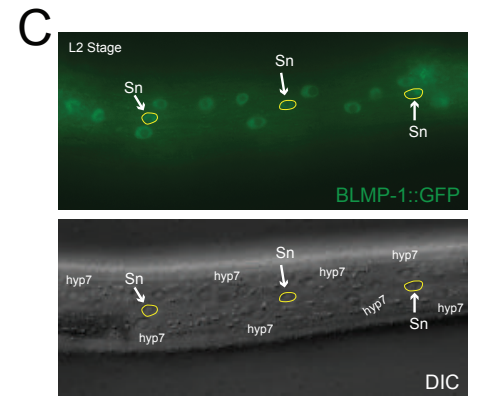
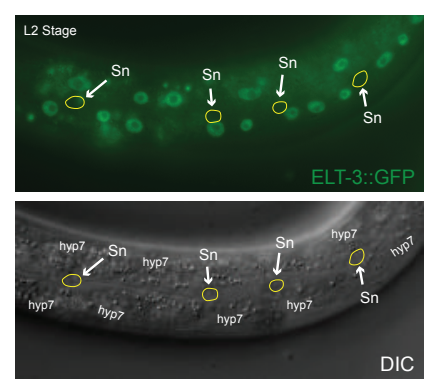

DIC

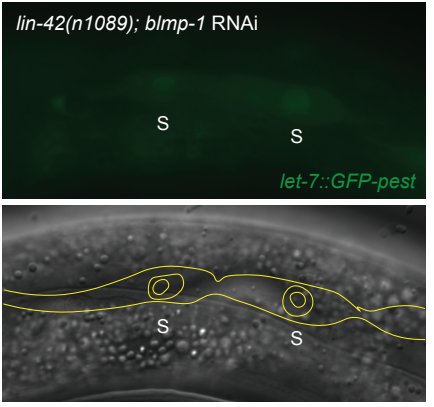

G

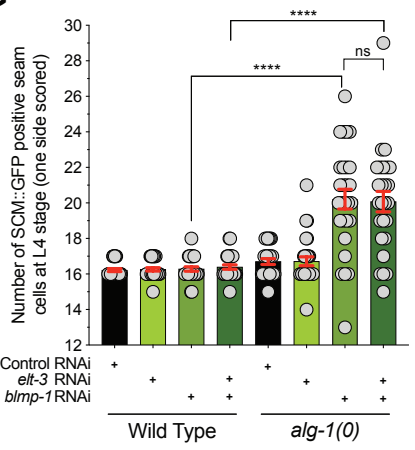

Stec et al. Figure 1 
A
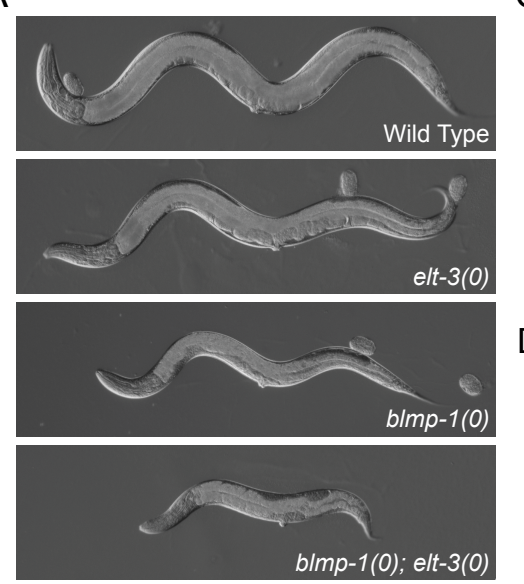

B

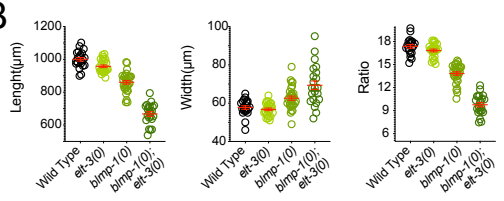

I

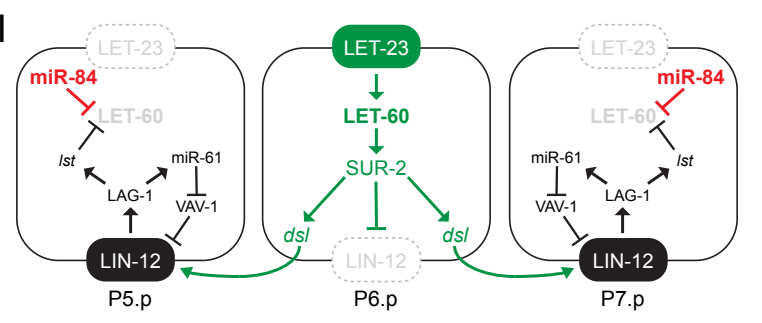

D
C
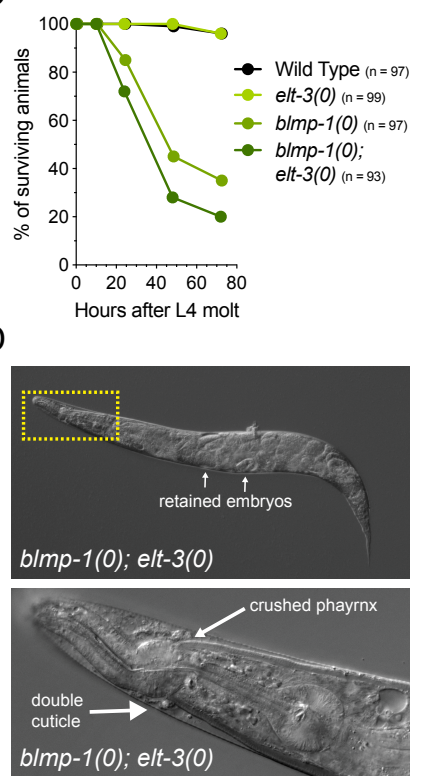

G

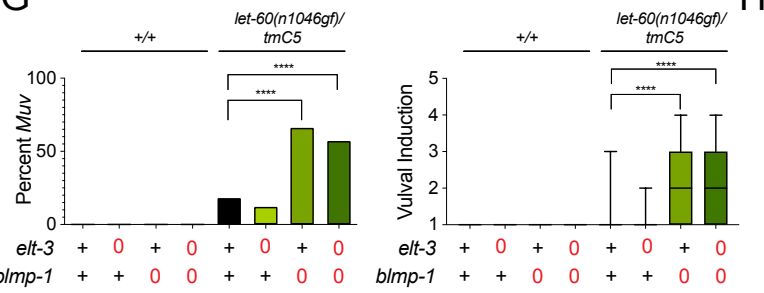

E
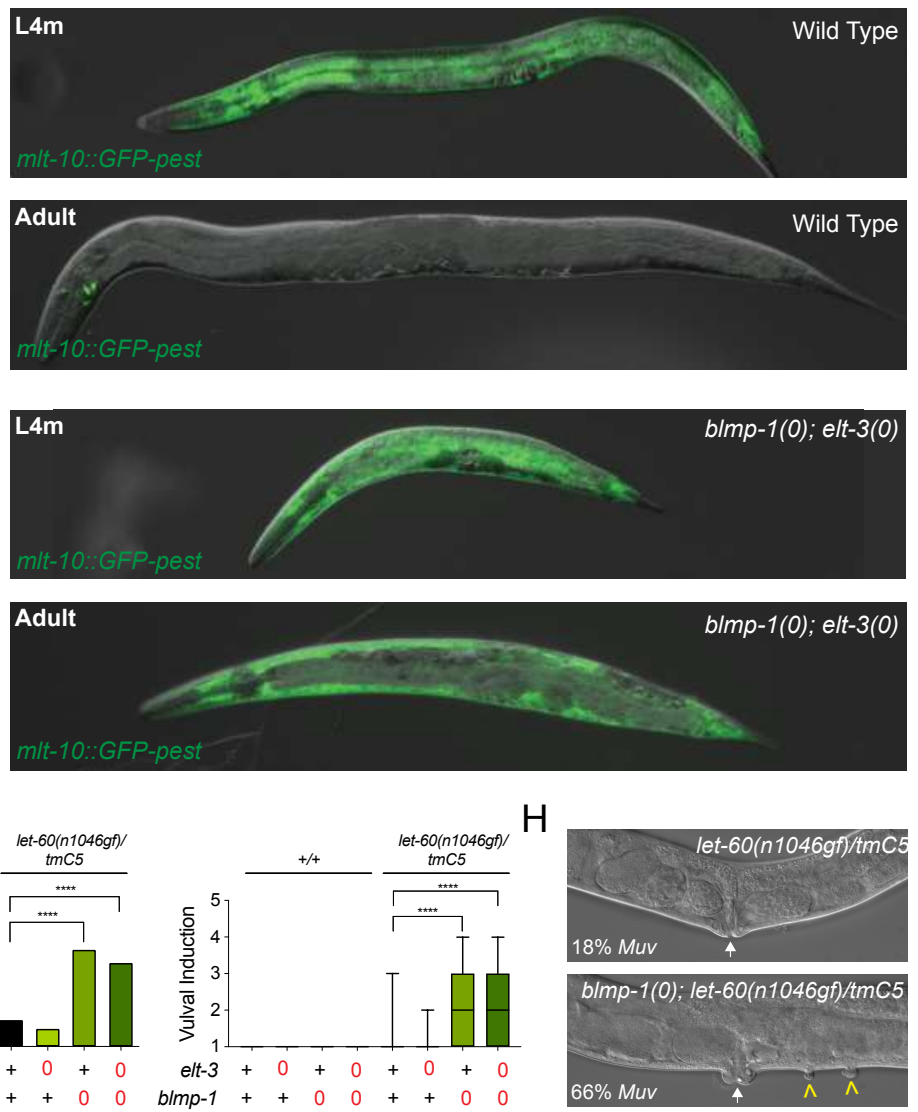

Stec et al. Figure 2 
A

B
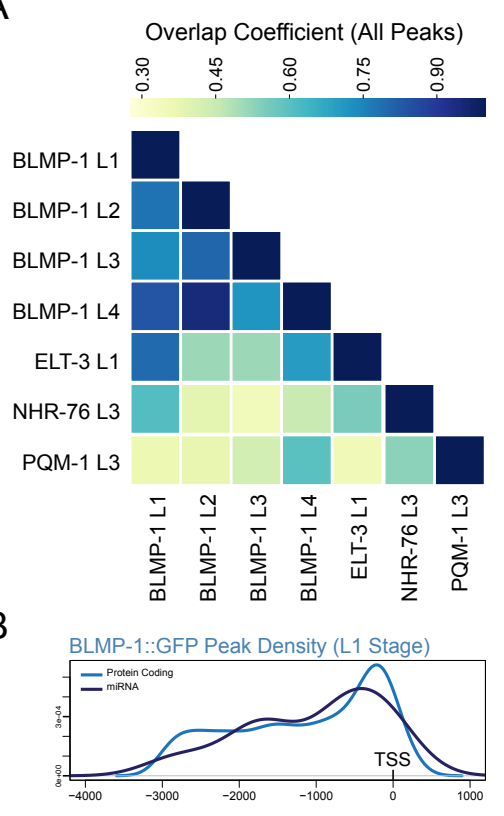

C
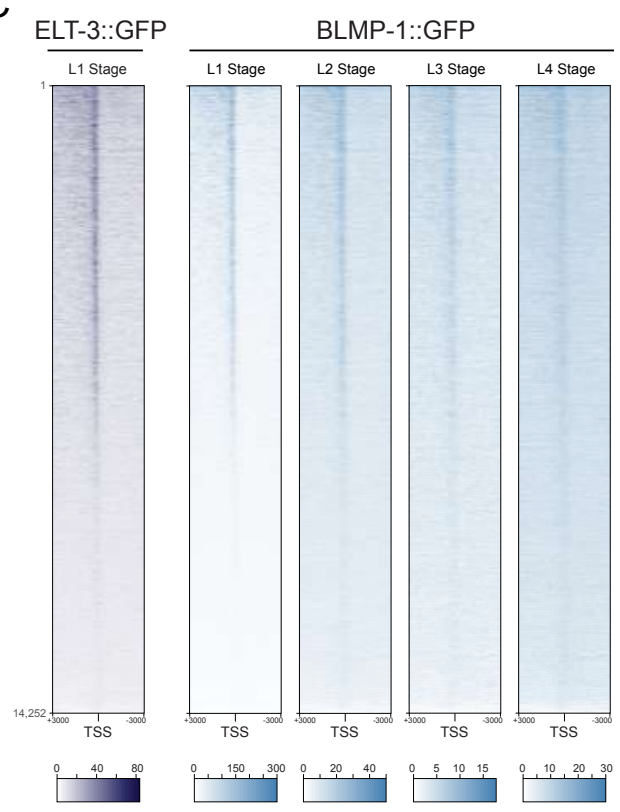

D

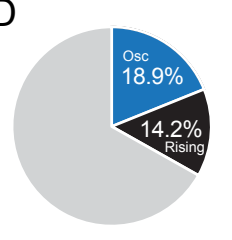

$\mathrm{E}$
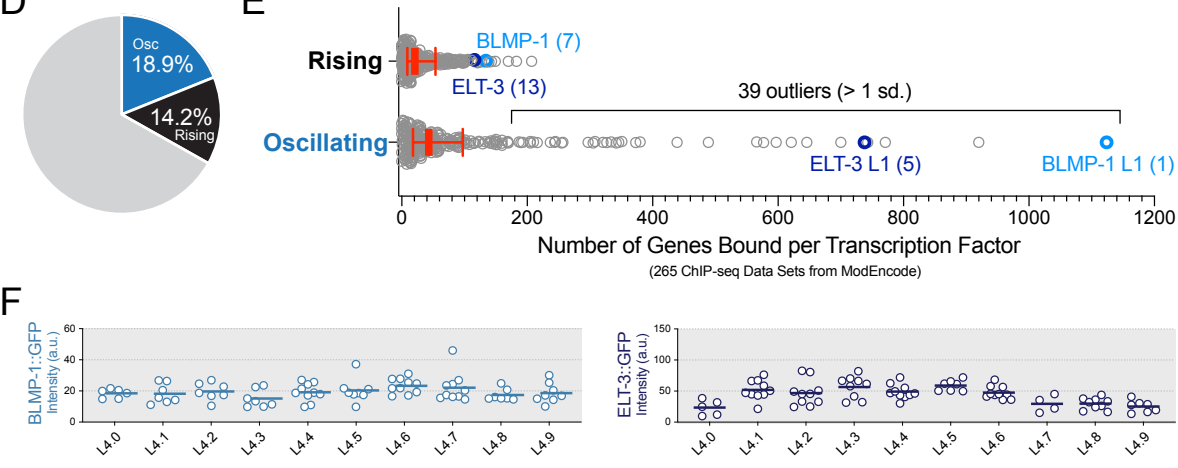

G
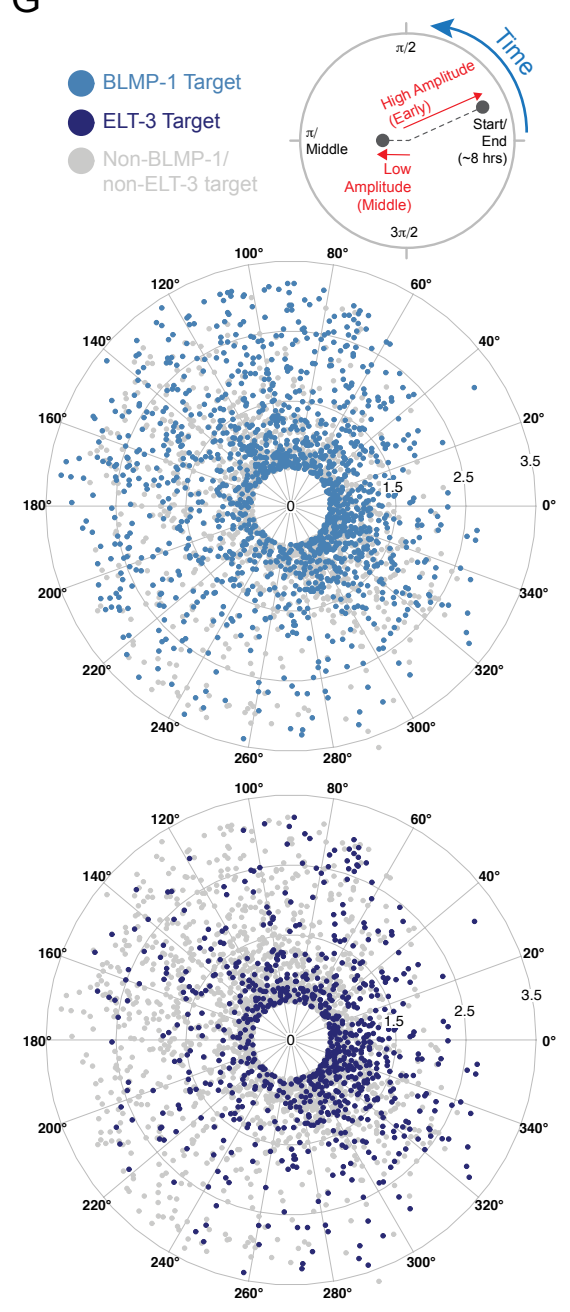

Stec et al. Figure 3 

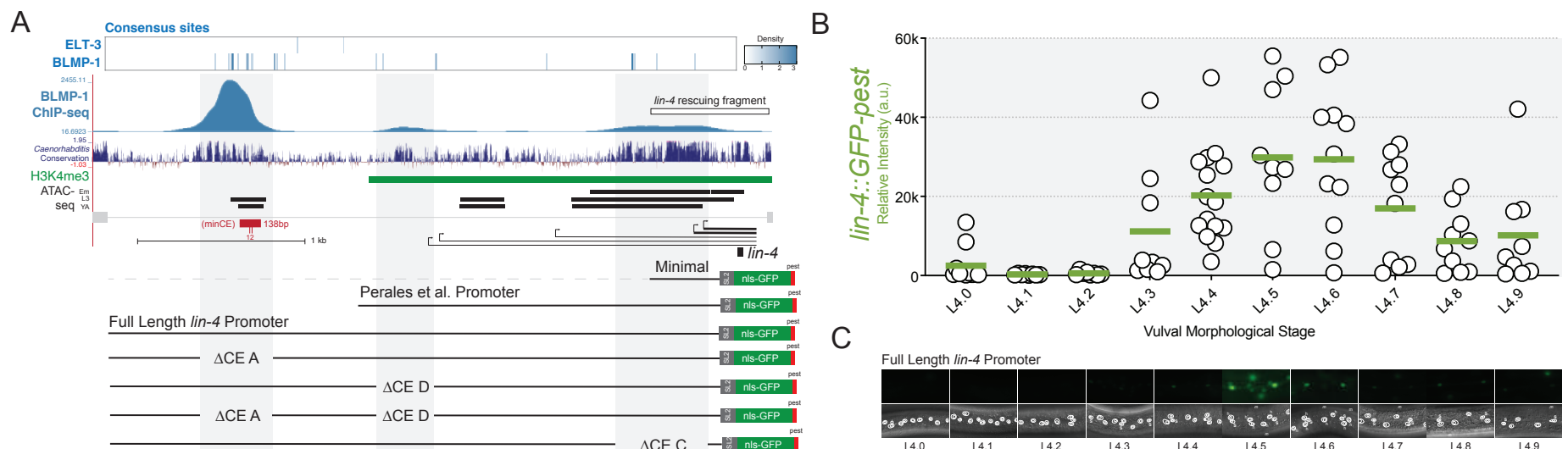

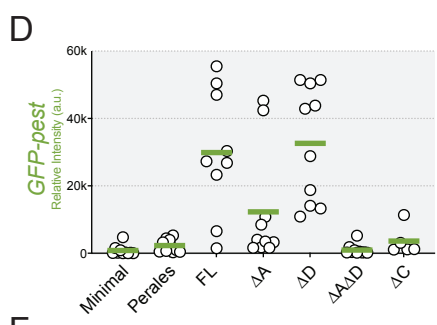

F

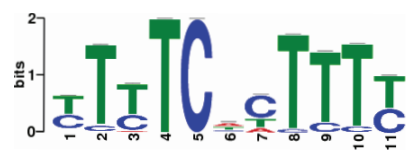

1 СТтTСТСТСТС - 3075bp

2 ттттстСттт - 2977ьp

E
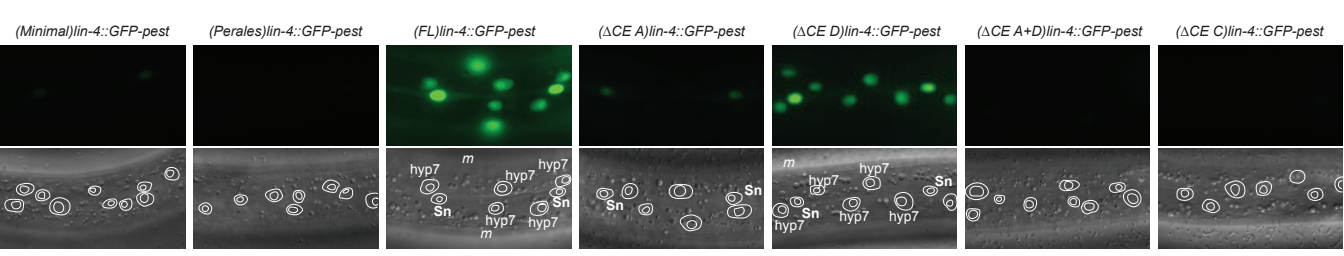

G

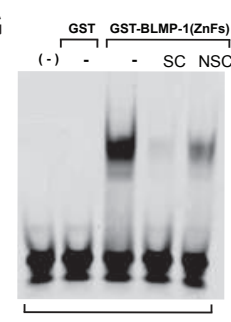

PCE probe 1

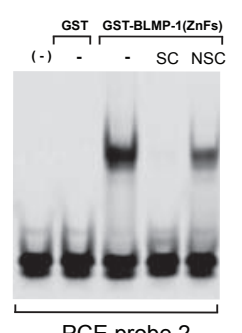

PCE probe 2
$\mathrm{H}$
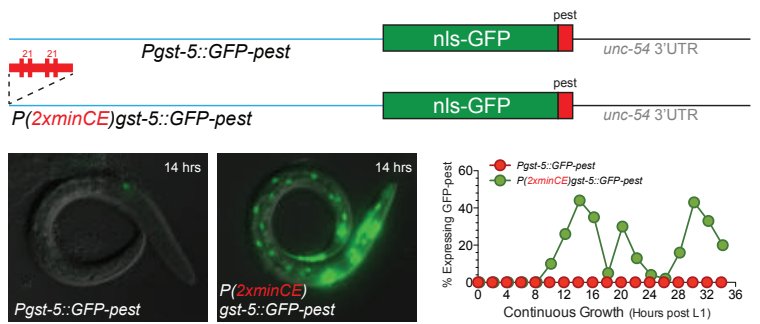

Stec et al. Figure 4 
bioRxiv preprint doi: https://doi.org/10.1101/2020.09.01.278127; this version posted September 3, 2020. The copyright holder for this preprint (which was not certified by peer review) is the author/funder. All rights reserved. No reuse allowed without permission.

A
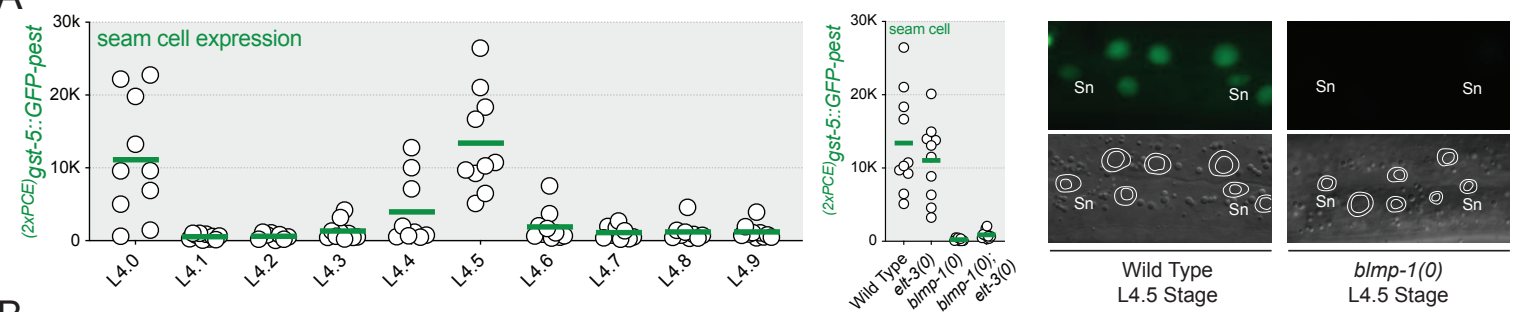

B
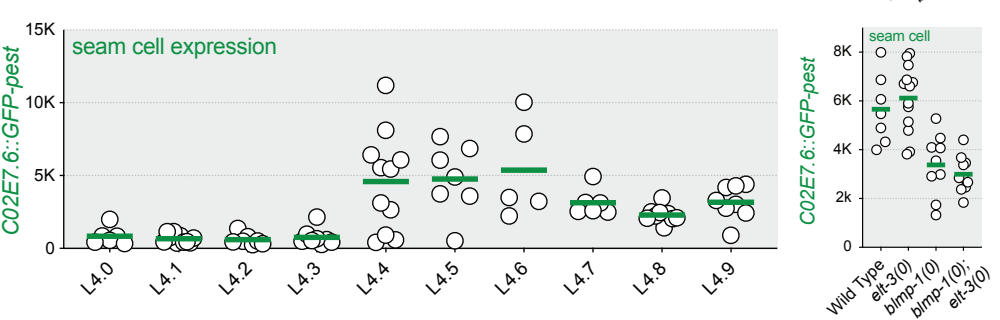

L4.5 Stage
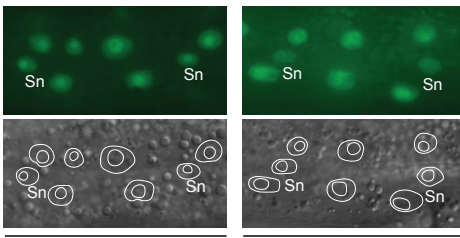

W4.5 Stage

blmp-1(0)

C
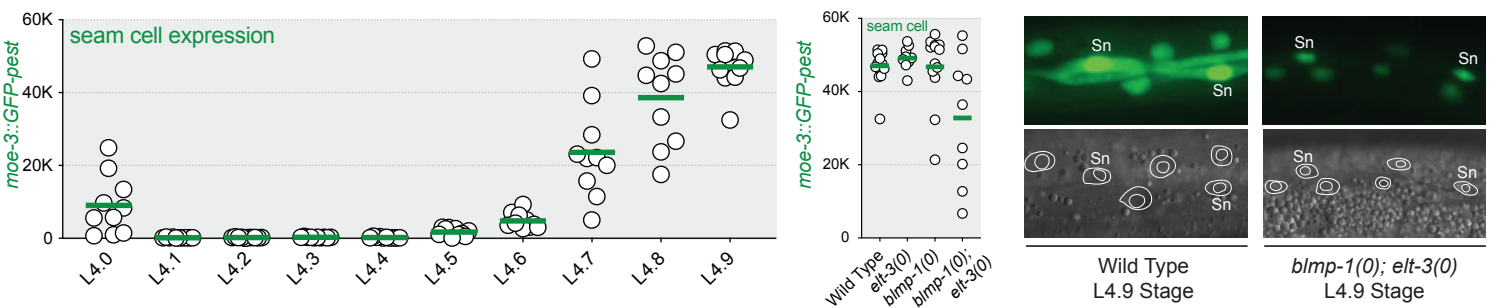

D

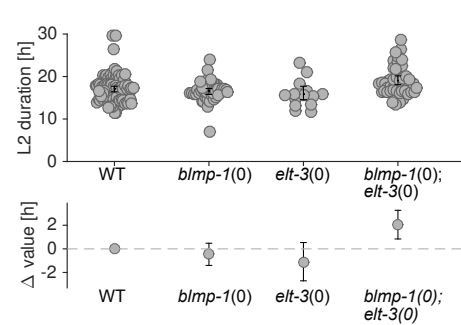

E
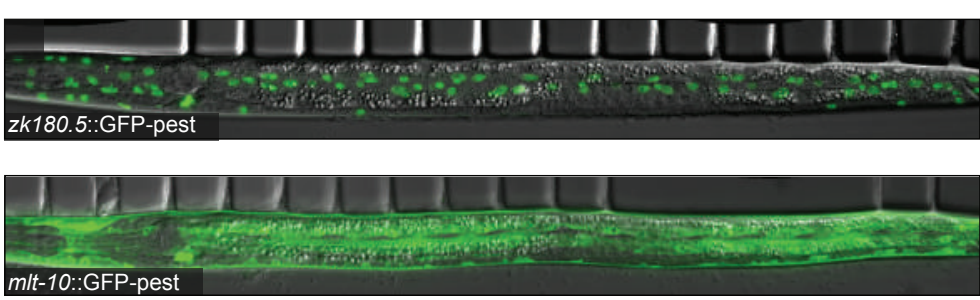

$\mathrm{F}$

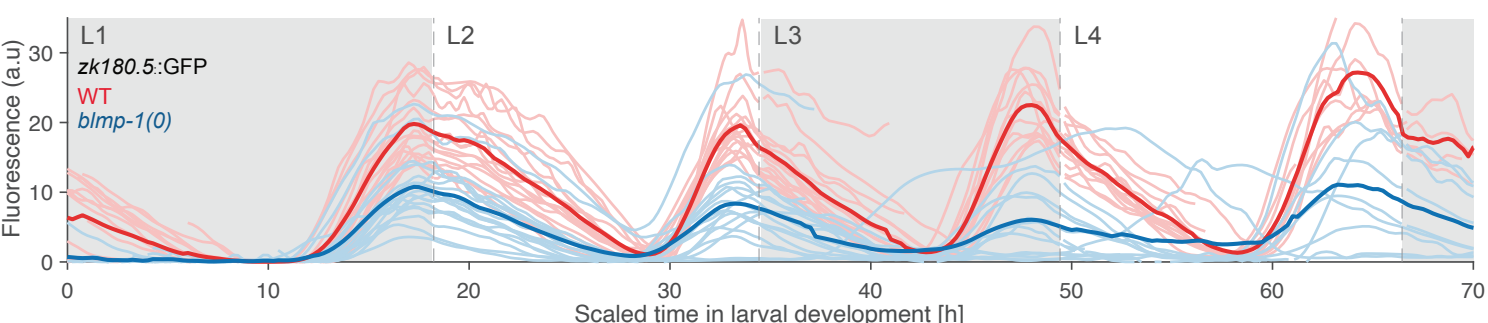

G
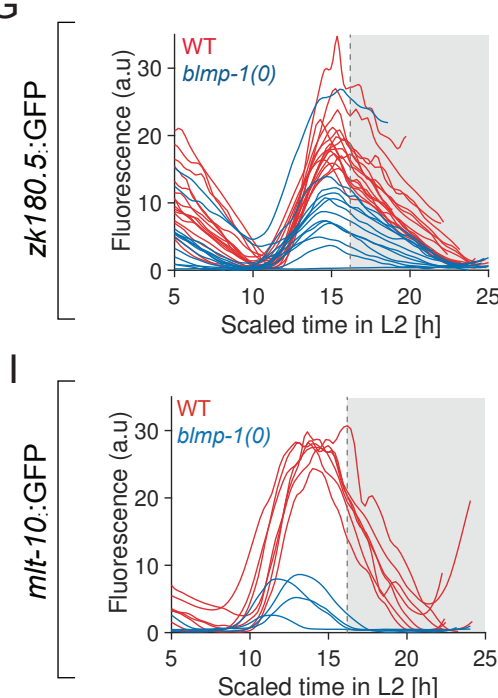
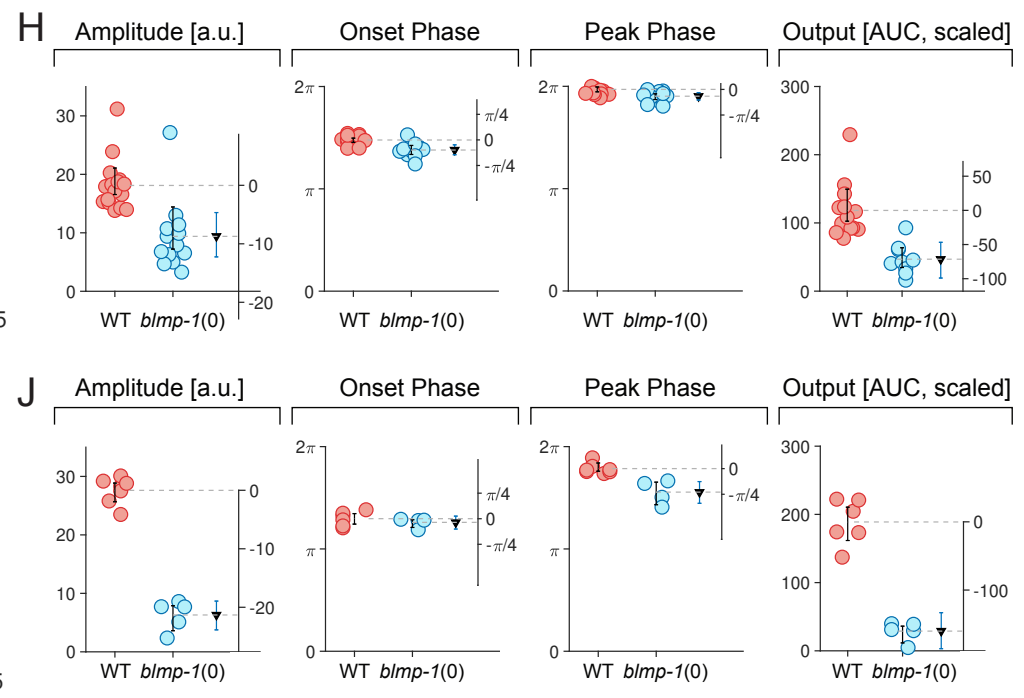

Stec et al. Figure 5 
A

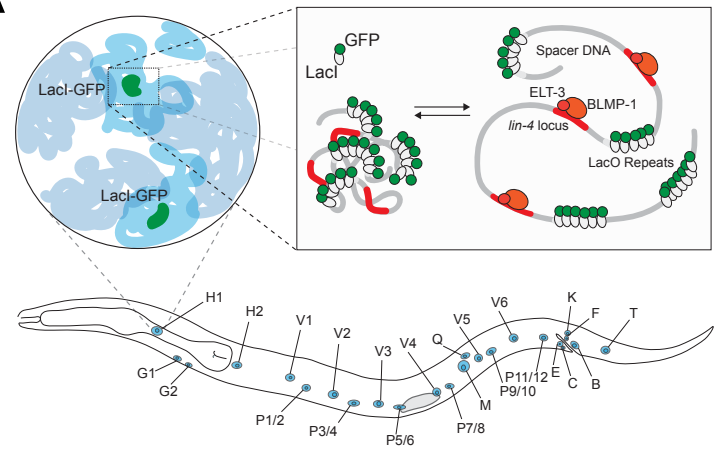

$\mathrm{B}$

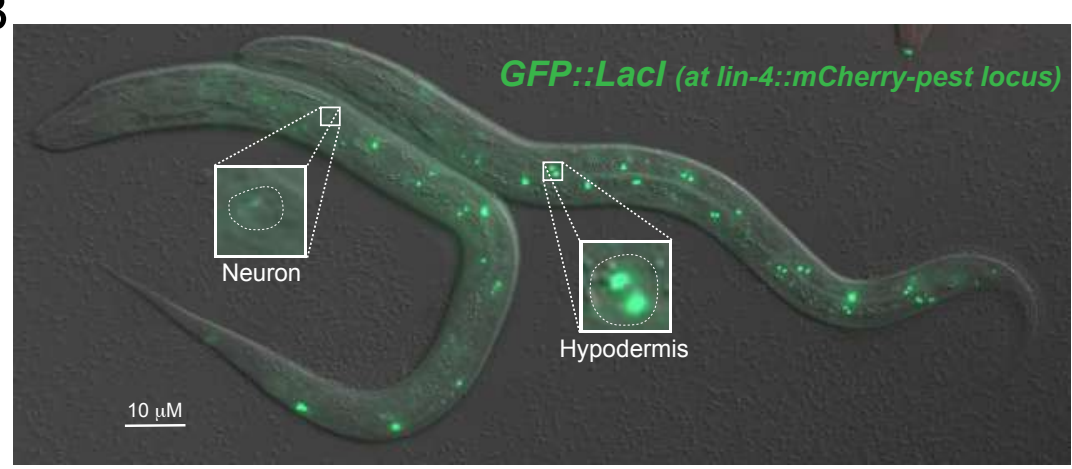

$\mathrm{C}$
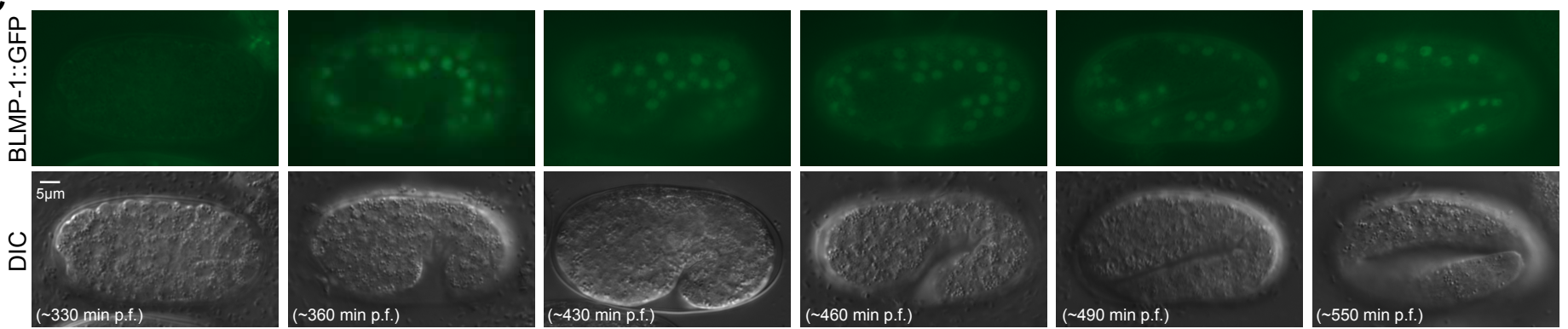

D

Wild Type
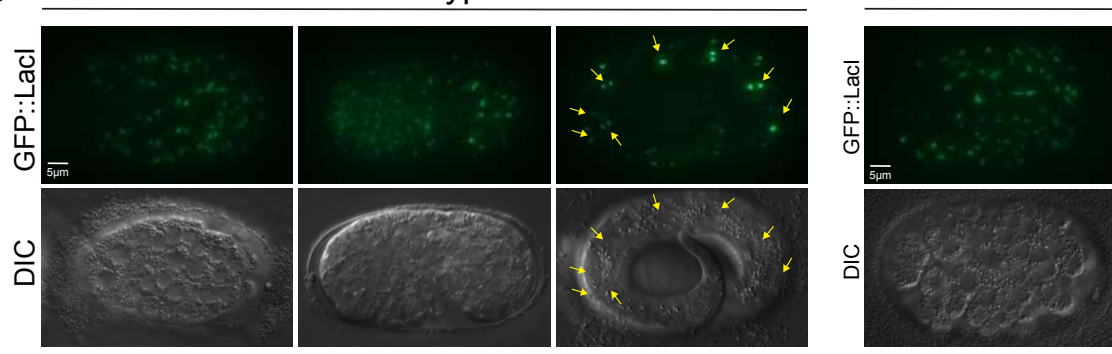

blmp-1(0)

$E$
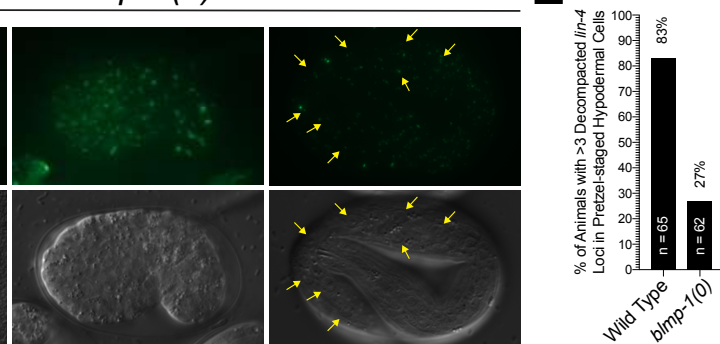

$\mathrm{F}$

Wild Type
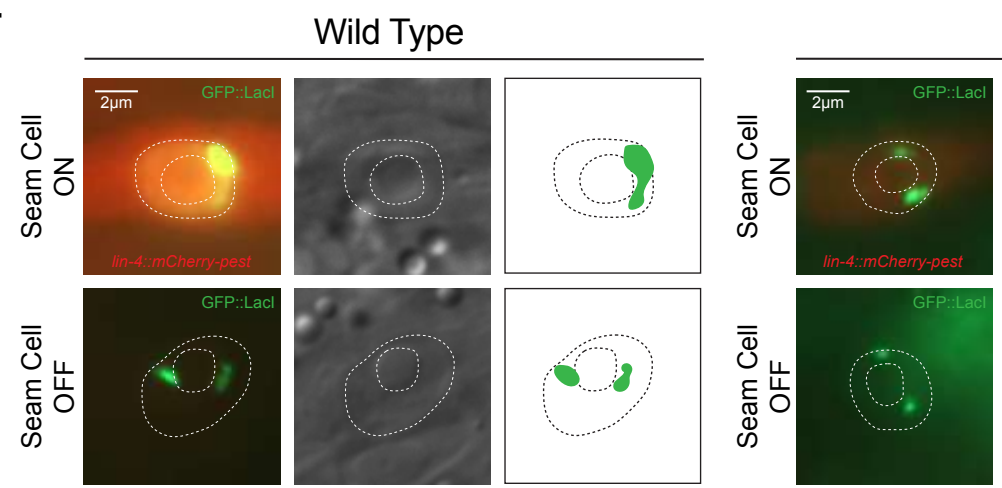

$b / m p-1(0)$
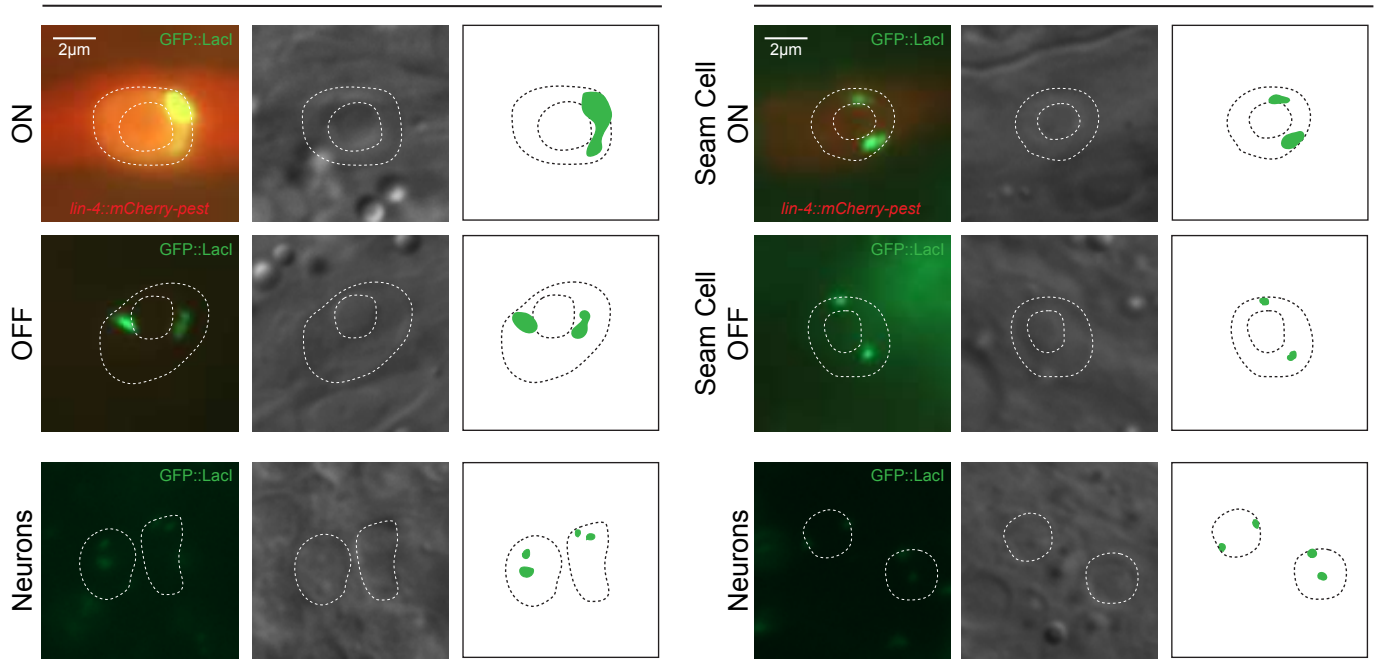

G

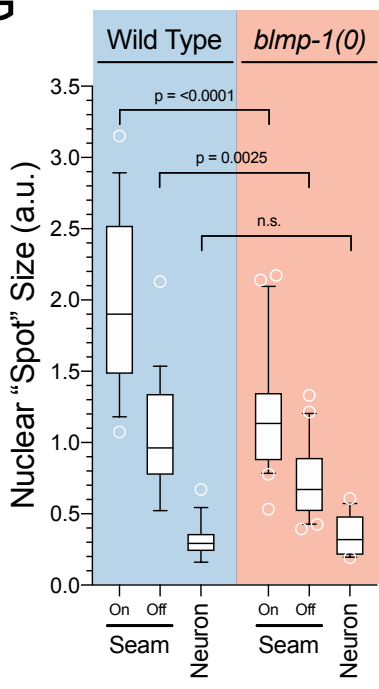

Stec et al. Figure 6 
A Grow from Hatching
for $\sim 22 \mathrm{hrs}$ at $20^{\circ} \mathrm{C}$

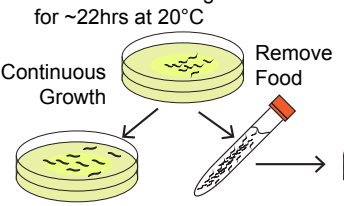

B

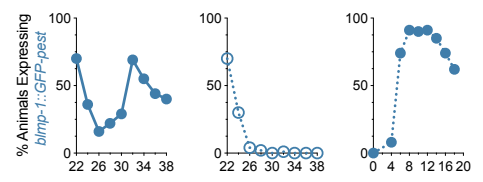

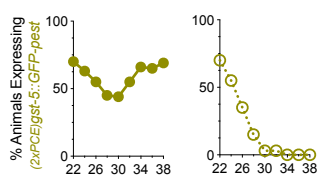

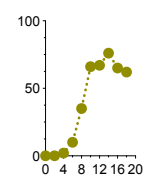

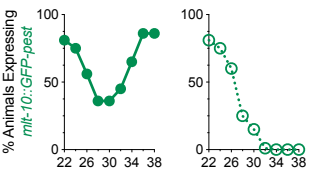
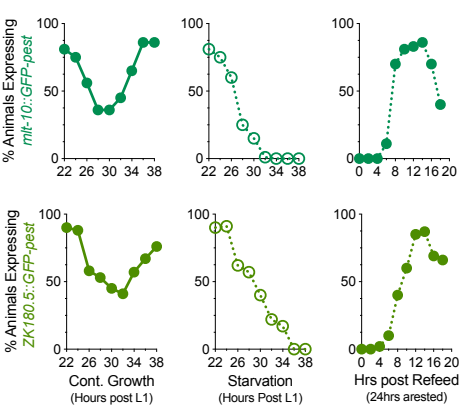
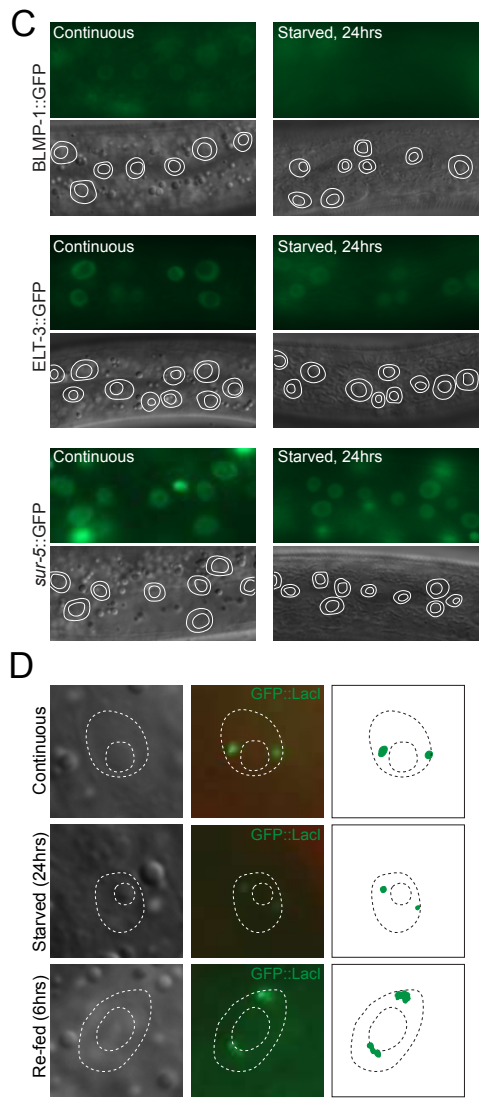

$\mathrm{H}$
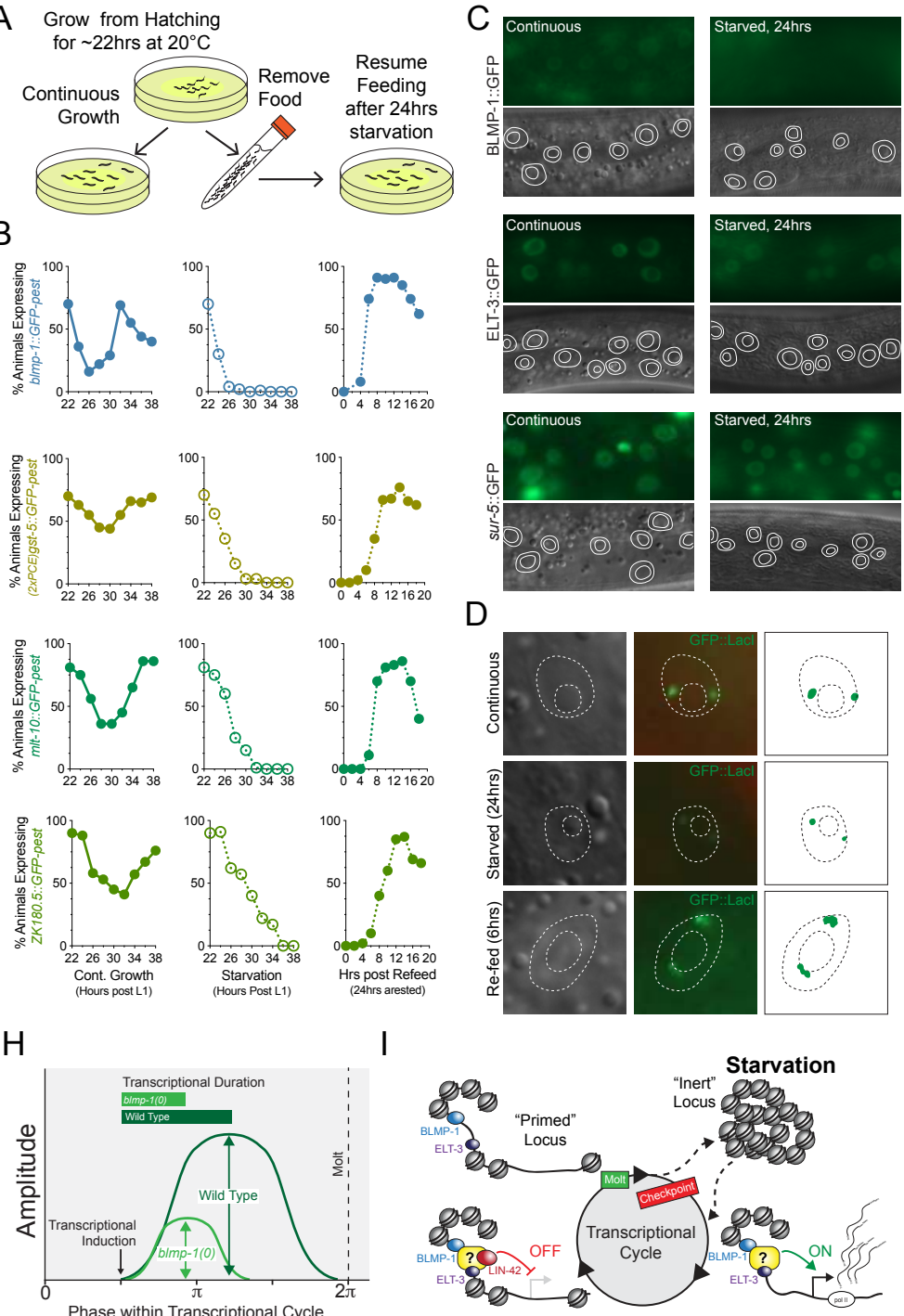
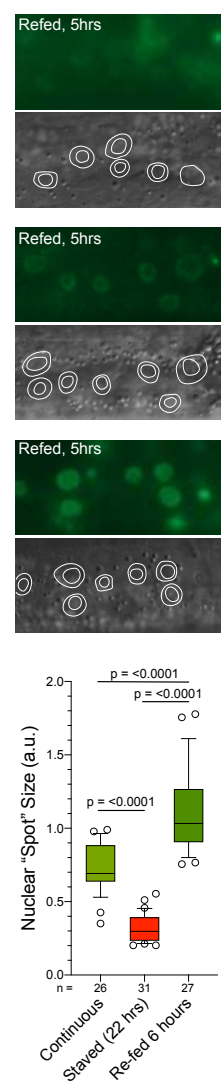

$\mathrm{J}$

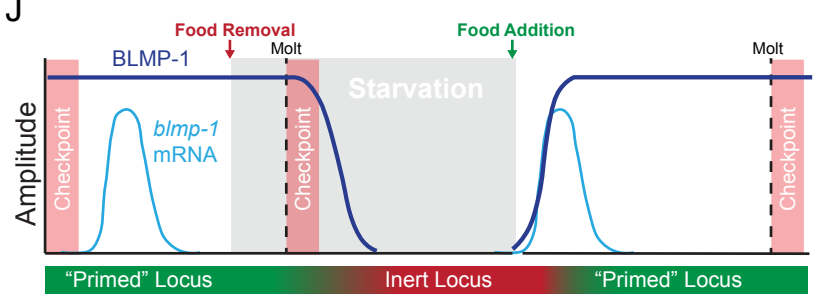

E Grow from Hatching for $\sim 22 \mathrm{hrs}$ at $20^{\circ} \mathrm{C}$

Continuous Remove

Growth Food

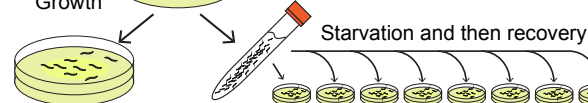

$\mathrm{F}$

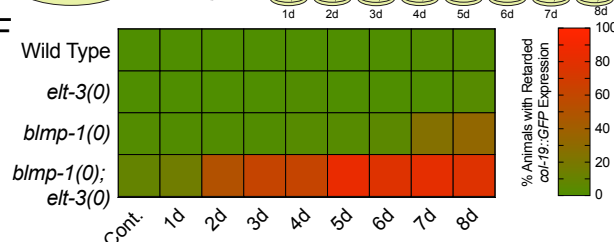

G
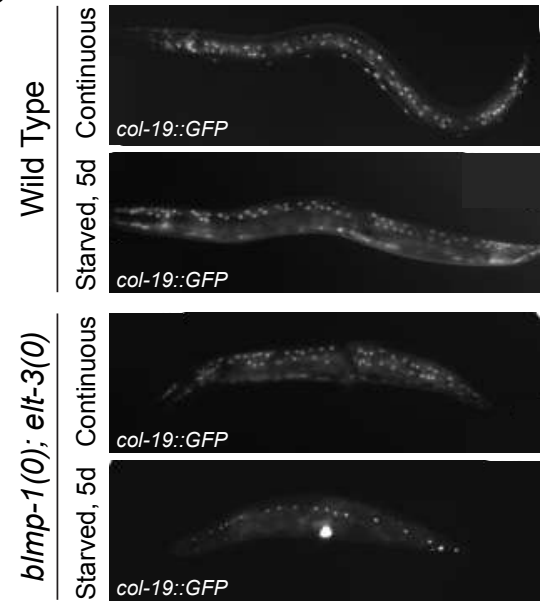

Stec et al. Figure 7 
A

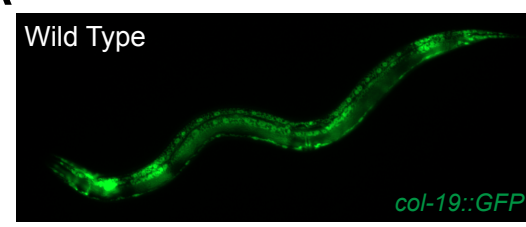

blmp-1(tm548); ain-1(ku322)

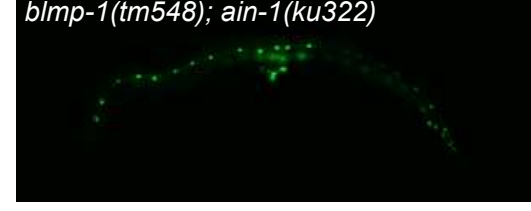

B

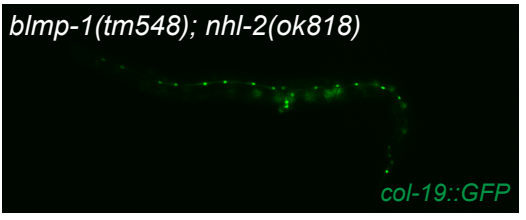

blmp-1(tm548); let-7(mg279)

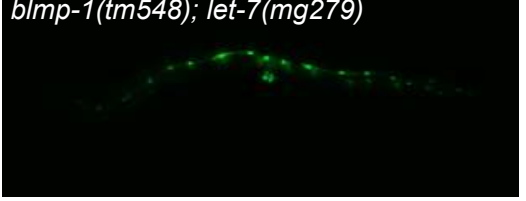

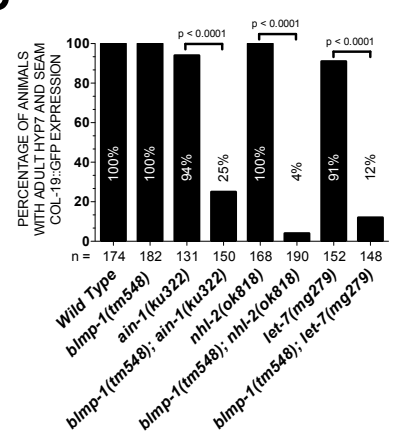

Stec et al. Supplemental Figure 1 
bioRxiv preprint doi: https://doi.org/10.1101/2020.09.01.278127; this version posted September 3, 2020. The copyright holder for this preprint (which was not certified by peer review) is the author/funder. All rights reserved. No reuse allowed without permission.
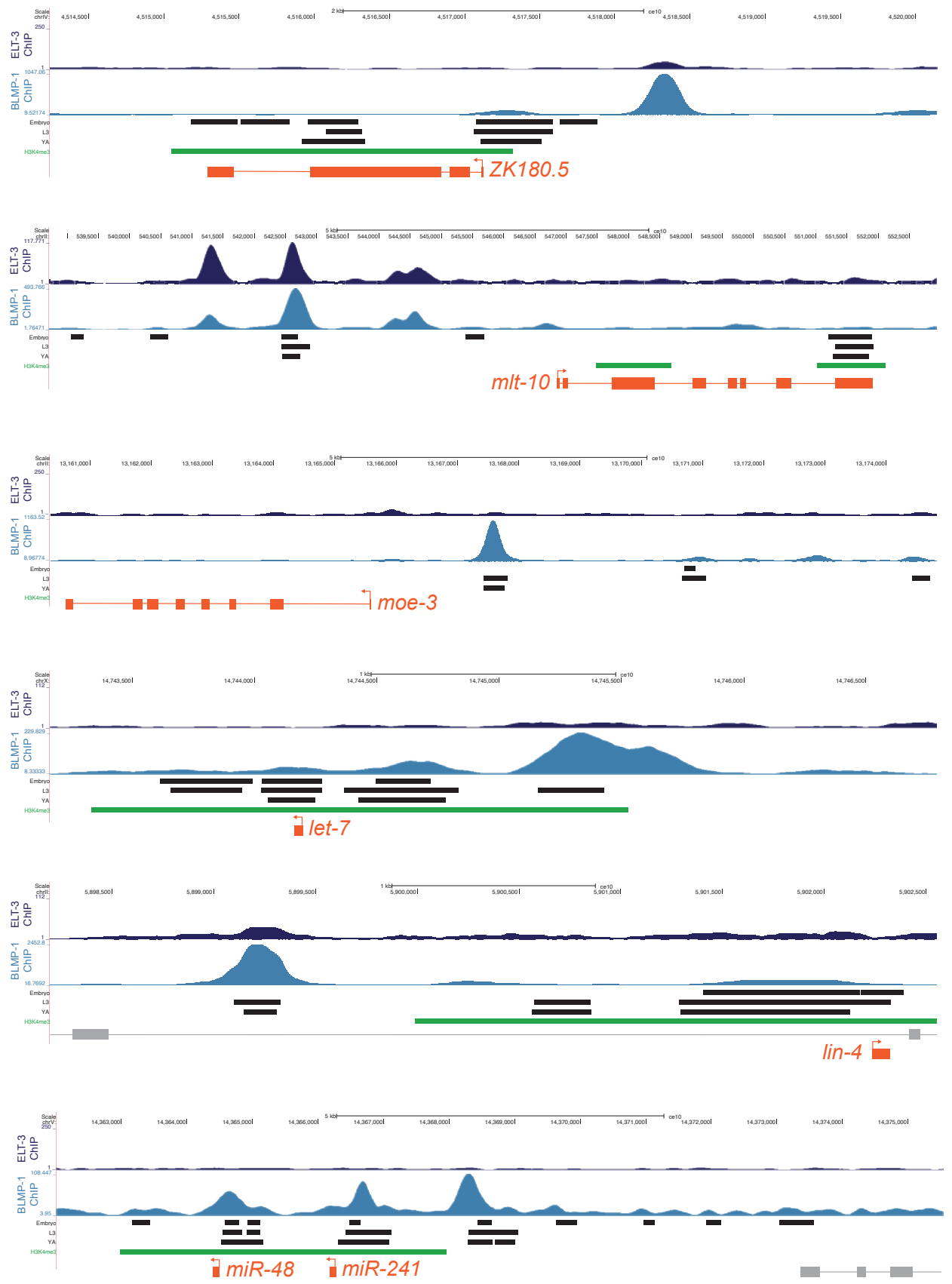

Stec et al. Supplemental Figure 2 
bioRxiv preprint doi: https://doi.org/10.1101/2020.09.01.278127; this version posted September 3, 2020. The copyright holder for this preprint (which was not certified by peer review) is the author/funder. All rights reserved. No reuse allowed without permission.

A

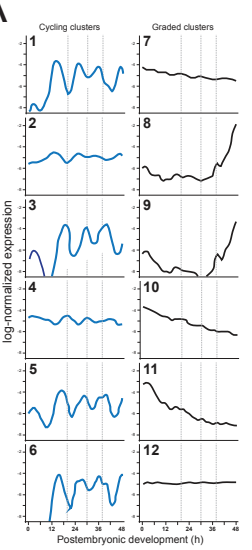

B

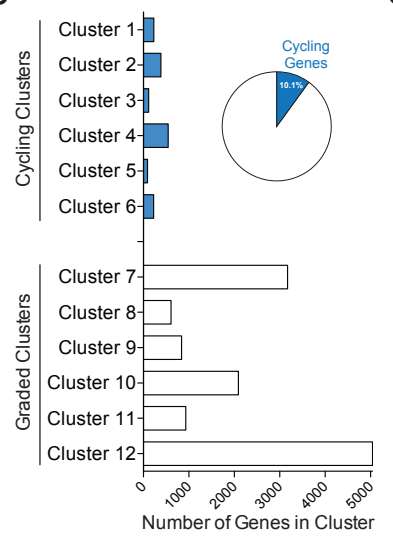

C

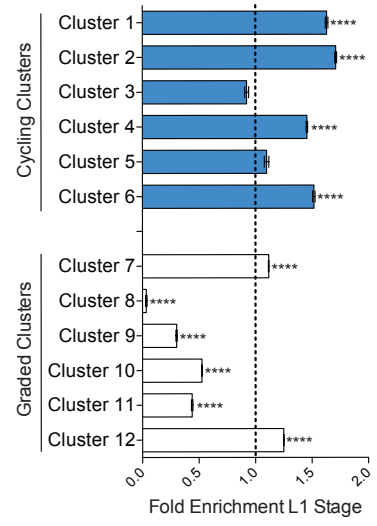

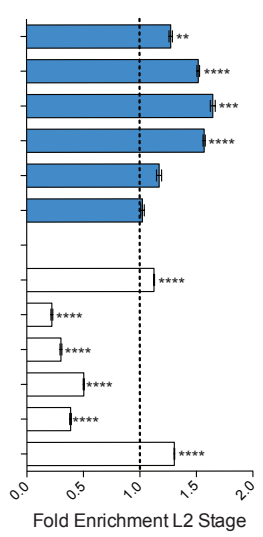

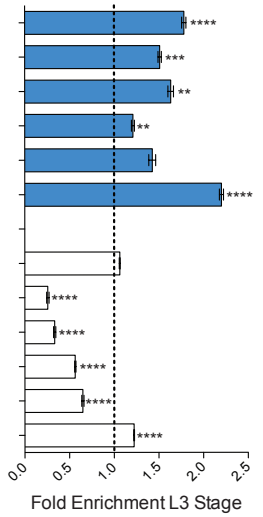

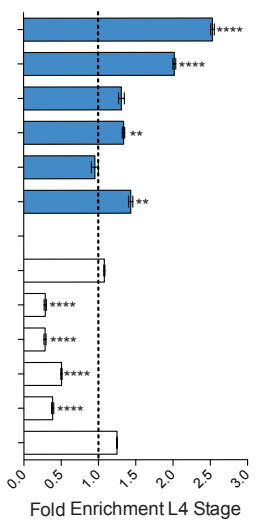

Stec et al. Supplemental Figure 3 
bioRxiv preprint doi: https://doi.org/10.1101/2020.09.01.278127; this version posted September 3, 2020. The copyright holder for this preprint (which was not certified by peer review) is the author/funder. All rights reserved. No reuse allowed without permission.

A
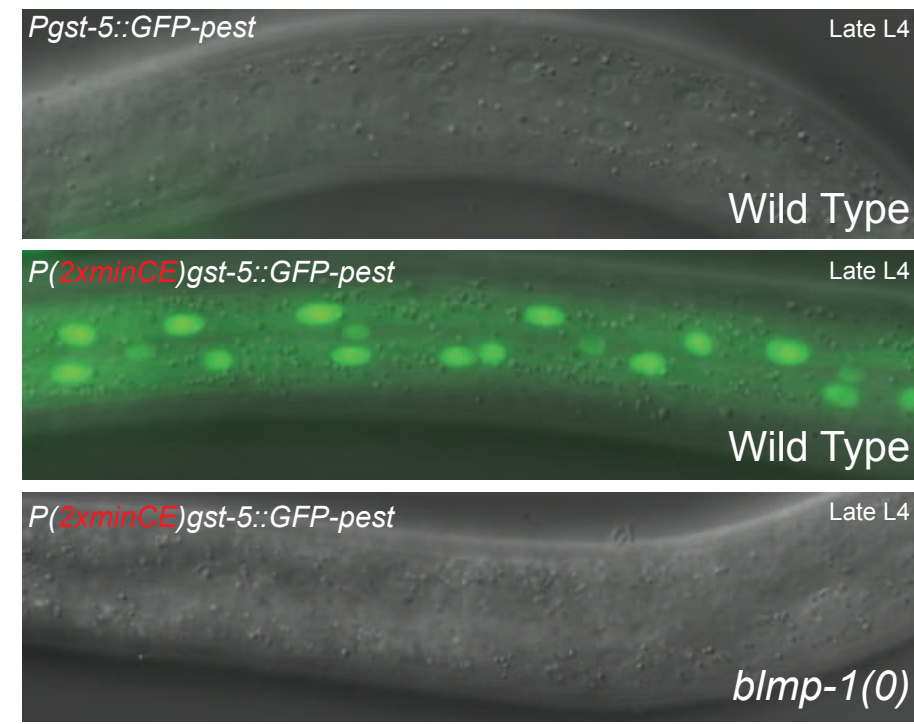

B

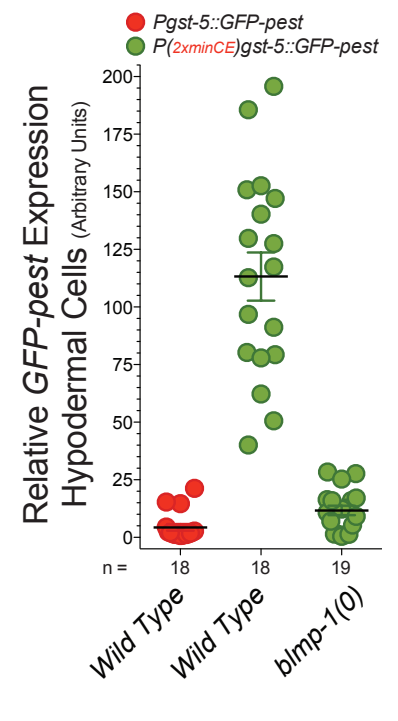

Stec et al. Supplemental Figure 4 

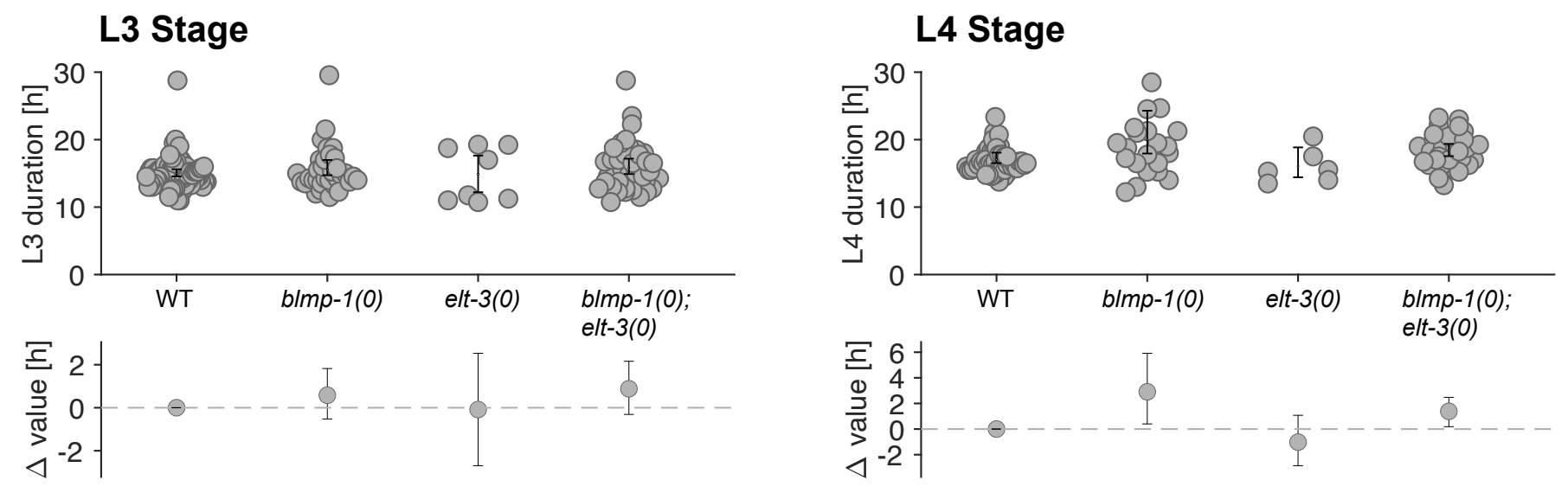

Stec et al. Supplemental Figure 5 
bioRxiv preprint doi: https://doi.org/10.1101/2020.09.01.278127; this version posted September 3, 2020. The copyright holder for this preprint (which was not certified by peer review) is the author/funder. All rights reserved. No reuse allowed without permission.

A

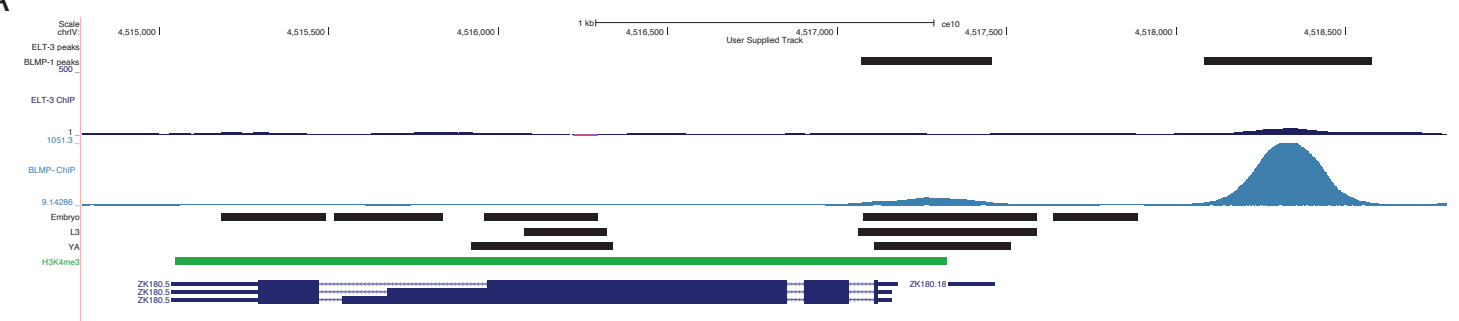

$\mathrm{B}$
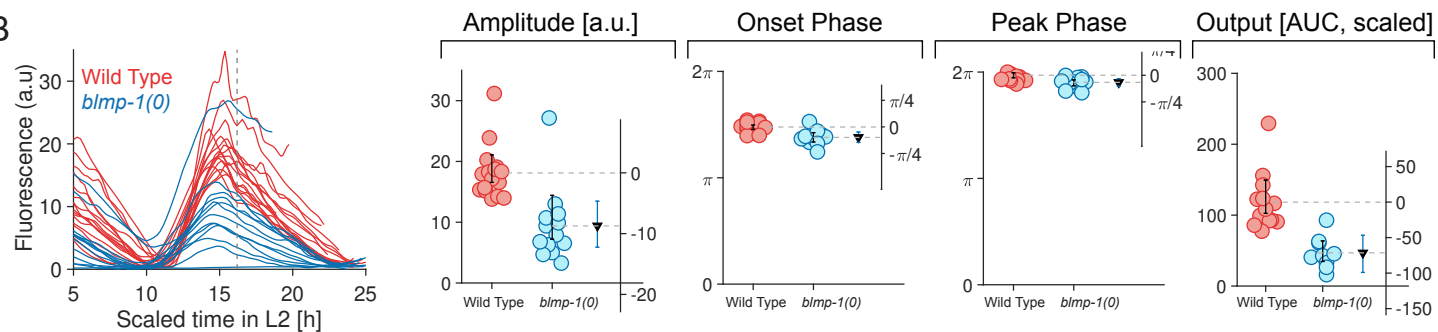

C
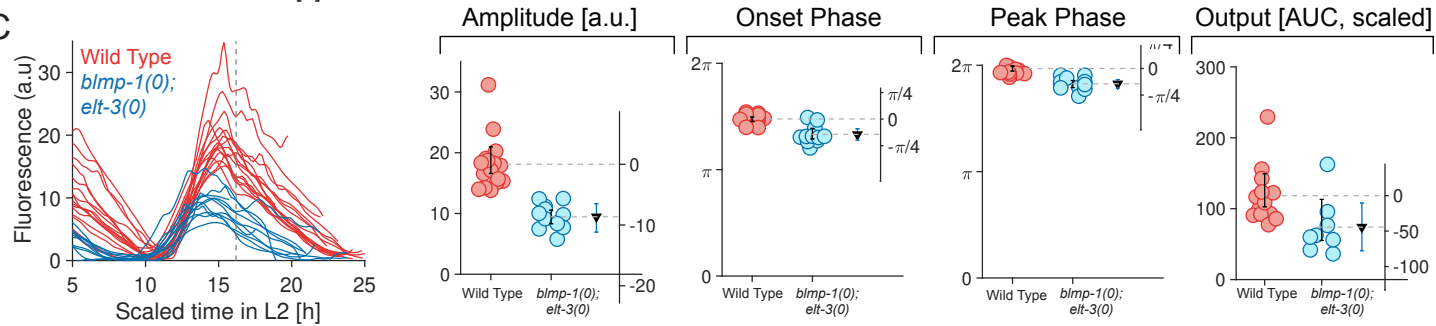

D

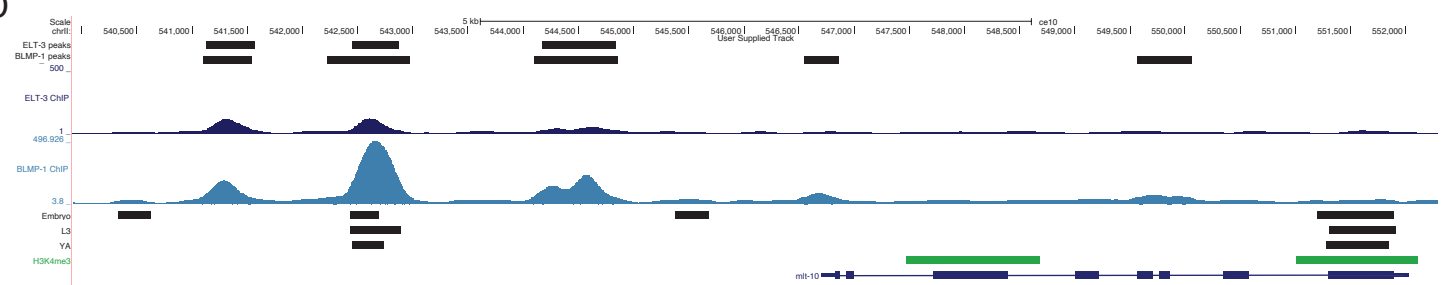

E

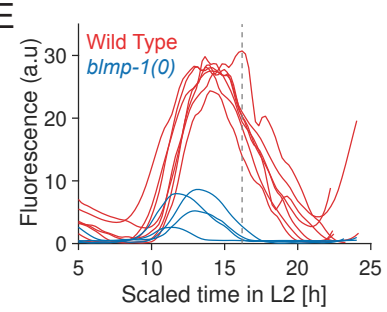

$\mathrm{F}$

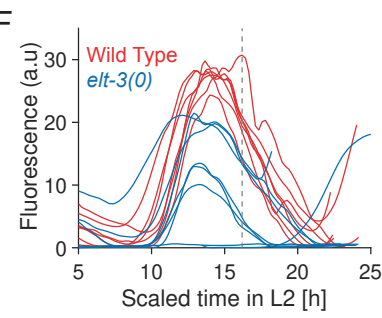

G

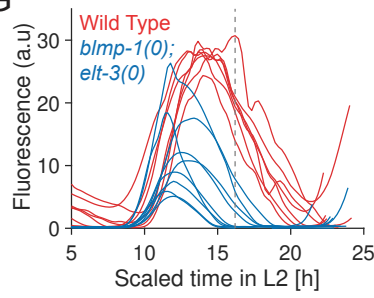

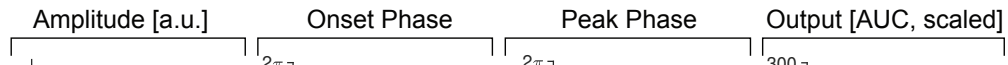

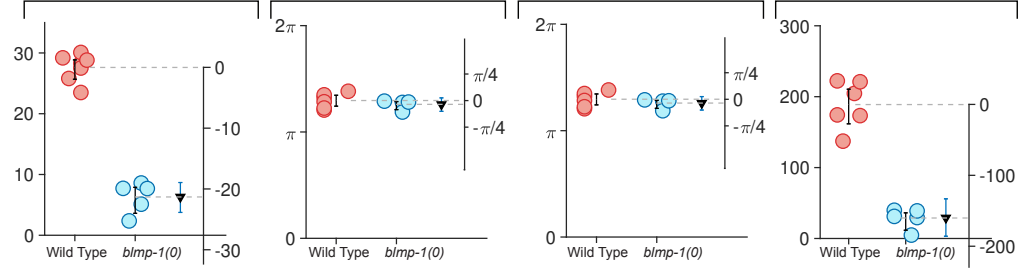

Amplitude [a.u.] $\quad$ Onset Phase Peak Phase Output [AUC, scaled]
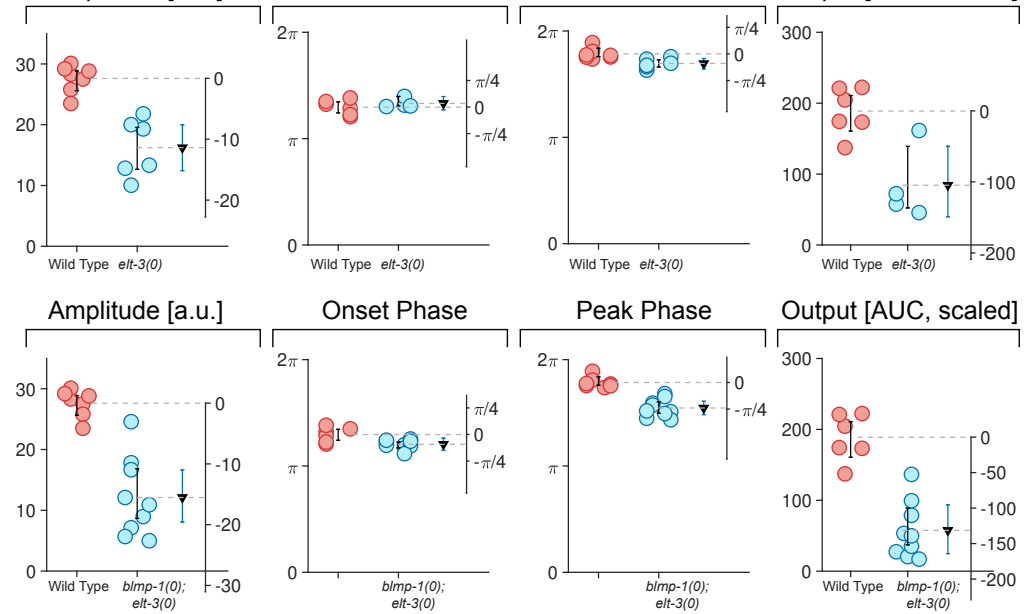
A

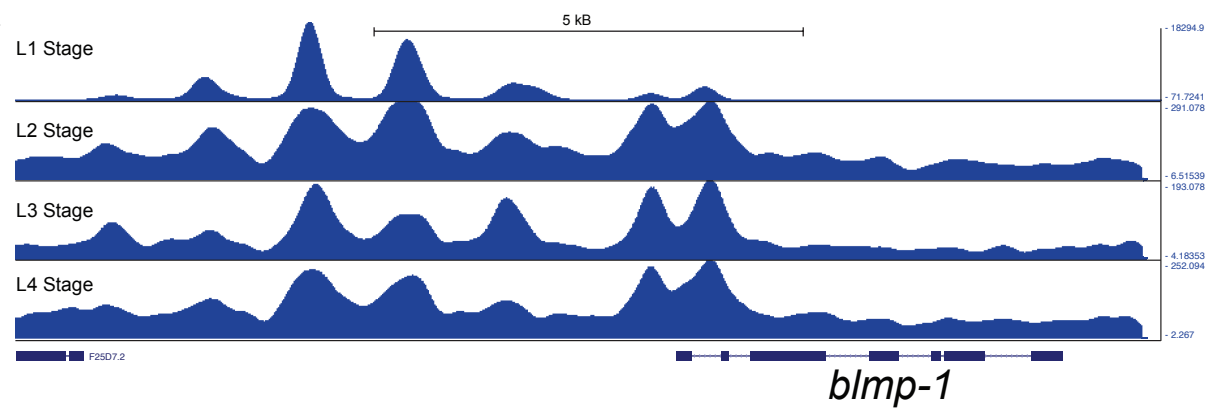

B

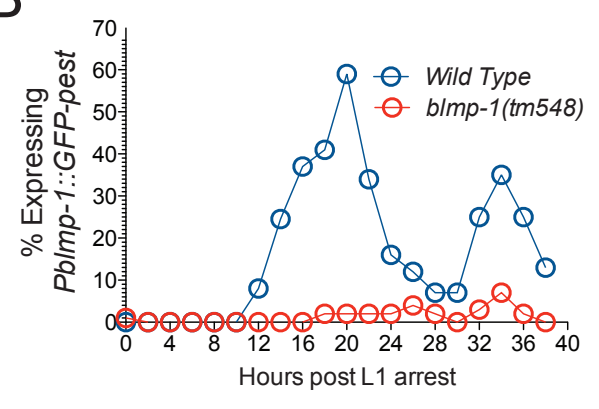

Stec et al. Supplemental Figure 7 
bioRxiv preprint doi: https://doi.org/10.1101/2020.09.01.278127; this version posted September 3, 2020. The copyright holder for this preprint (which was not certified by peer review) is the author/funder. All rights reserved. No reuse allowed without permission.

A
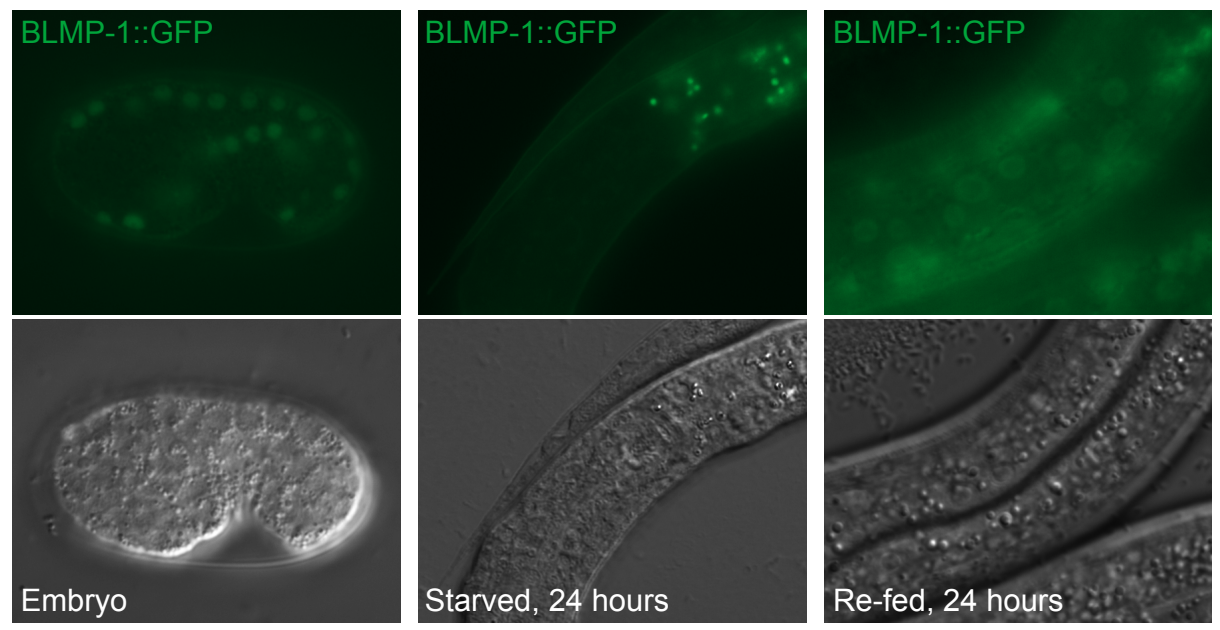

B

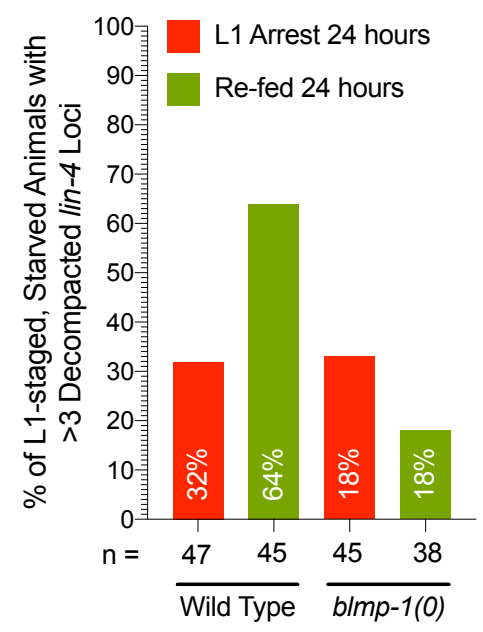

Stec et al. Supplemental Figure 8 